\title{
Dynamics of bubbles under stochastic pressure forcing
}

\author{
Riccardo Vesipa, Costantino Manes, and Luca Ridolfi \\ Department of Environment, Land and Infrastructure Engineering, \\ Politecnico di Torino, C.so Duca degli Abruzzi 24, 10129 Torino, Italy* \\ Eleonora Paissoni \\ Cranfield Water Science Institute, Cranfield University, Bedfordshire, MK43 OAL, UK
}

\begin{abstract}
Several studies have investigated the dynamics of a single spherical bubble at rest under a nonstationary pressure forcing. However, attention has almost always been focused on periodic pressure oscillations, neglecting the case of stochastic forcing. This fact is quite surprising, as random pressure fluctuations are widespread in many applications involving bubbles (e.g., hydrodynamic cavitation in turbulent flows or bubble dynamics in acoustic cavitation) and noise, in general, is known to induce a variety of counter-intuitive phenomena, in non-linear dynamical systems such as bubble oscillators. In order to shed light on this unexplored topic, here we study bubble dynamics as described by the Keller Miksis equation, under a pressure forcing described by a Gaussian colored noise modeled as an Ornstein-Uhlenbeck process. Results indicate that, depending on noise intensity, bubbles display two peculiar behaviors: when intensity is low, the fluctuating pressure forcing mainly excites the free oscillations of the bubble, and the bubble's radius undergoes small amplitude oscillations with a rather regular periodicity. Differently, high noise intensity induces chaotic bubble dynamics, whereby non linear effects are exacerbated and the bubble behaves as an amplifier of the external random forcing.
\end{abstract}

Over the last decades, the dynamics of gas-bubbles ${ }^{33}$ (also referred to as cavities) in liquids has attracted a ${ }^{34}$ lot of interest in the scientific community [e.g., 1-3]. 35 This paper focuses on the canonical case of a spherical 36 bubble subjected to a prescribed external forcing which 37 drives variations in the bubble's radius. The problem 38 has been extensively addressed [e.g., see 4-7] and can be 39 mathematically described by ordinary differential equa- 40 tions, which, depending upon different simplifying as- 41 sumptions, can take different forms [8-12]. Despite such 42 differences, all these equations share the common feature 43 of retaining strongly non-linear terms which make gas- 44 bubbles in liquids dynamically-rich systems [13].

One of the attractive features of bubble dynamics in- 46 volves the possibility of cavities to undergo abrupt vari- 47 ations in size. In particular, due to the high inertia of 48 the liquid hosting the cavities, bubbles, if properly ex- 49 cited, can be subjected to abrupt collapses that generate ${ }_{50}$ intense pressure and temperature peaks, which, in turn, ${ }_{51}$ are associated with the generation of shock waves and 52 the emission of light and sound [14-16].

The attractiveness of such extreme pressure and tem- 54 perature events stems from the fact that they can be 55 exploited in several technological applications. For in- 56 stance, in medicine, bubble collapses are used to break 57 liver and kidney stones and cancer cells $[17,18]$. In the 58 water industry, bubbles' collapses physically inactivate 59 bacteria and the free-radicals generated by the tempera- 60 ture peaks reached during the collapsing phase are used ${ }_{61}$

\footnotetext{
* riccardo.vesipa@polito.it
}

to oxidize pollutants for waste-water treatment purposes [19-22]; in geophysics, bubble implosions are useful for sub-sea geological explorations [23, 24].

Several factors influence bubble dynamics. The most relevant are the properties of the liquid hosting the gas bubble [25], the presence of solid boundaries close to the bubble [26-28], the interaction with other proximal gas cavities $[29,30]$, and the action of an external forcing that alters the bubble equilibrium conditions. Two classes of forcing are commonly considered. The first one consists in the alteration of the bubble size in a liquid at rest (with time-invariant pressure) using either laser beams or sparks [31,32]. The second class involves variations of the static pressure of the liquid hosting the bubble $[33,34]$.Static pressure variations are usually induced by ultrasound waves traveling within a volume of liquid at rest $[35,36]$ or by alterations of the liquid velocity (e.g., geometrical constrictions like orifice plates or Venturi tubes) in a pressurized system of conduits [37, 38].

The pressure forcing - especially, the case of pressure fluctuations in a liquid at rest - has been the focus of a great deal of studies and will be considered also in the present paper. The largest part of previous works have generally explored the effects of sinusoidal pressure oscillations on the bubble's radius [e.g., 39]. In spite of the simple and regular temporal structure of the forcing, the response of the bubble turned out to be very rich, exhibiting period-doubling bifurcations and perioddoubling cascades that can ultimately lead to a chaotic behavior [40-45].

Other studies have investigated the forced dynamics of bubbles when the pressure of the hosting liquid is perturbed by a bi-harmonic signal obtained as the sum of two sinusoidal signals $[46,47]$. It was found that such 65 a combined signal induces significant alterations in the 
thresholds of period-doubling bifurcations and period- 99 doubling cascades. It was therefore suggested to adoptioo bi-harmonic pressure signals to control chaos inception 101 and to give a more controlled and predictable bubble ${ }_{102}$ behavior [48]. Finally, some theoretical and experimen-103 tal studies have focused on the transient phase occurring ${ }_{104}$ during the inception of an ultrasound field and in pulsed ${ }_{105}$ ultrasound fields [49-51]. Results showed that the $\mathrm{col}_{-106}$ lapse of bubbles was more intense in the transient phases, ${ }_{107}$ rather than during the regular sinusoidal phase of pulsed ${ }_{108}$ ultrasound fields.

To the authors' opinion, the aforementioned results 110 from the literature suggest that transients and irregu ${ }_{111}$ larities of the external forcing can lead to yet unexplored ${ }_{112}$ bubbles' responses. This should not be entirely surpris-113 ing because it is well known that many interesting and ${ }_{114}$ unexpected phenomena emerge from the stochastic forc- ${ }_{115}$ ing (i.e., a form of irregular forcing) of strongly non-linear systems (i.e., the so called noise-induced phenomena, see [52-55]). It is within this context that the aim, novelty and relevance of the present paper are cast. The aim is in- ${ }^{11}$ deed to explore the response of a single bubble to random fluctuations of the external pressure. The study is novel ${ }^{117}$ because, to the best of the authors' opinion, it has never been addressed before. Its relevance lies in the fact that ${ }_{118}$ stochastic pressure-forcing are important for a number ${ }_{119}$ of applications and are encountered in a number of envi-120 ronments. Notable examples include: (i) hydrodynamic ${ }_{121}$ cavitation reactors (mainly used for water-treatment pro-122 cesses) where the pressure fluctuations imposed by tur-123 bulence and by the geometry of the reactor are known ${ }_{124}$ to heavily influence bubbles' dynamics $[30,43,56]$ and,125 ultimately, bubble's efficiency in oxidation and disinfec-126 tion processes; and (ii) acoustic cavitation reactors where bubbles' dynamics is influenced by the interactions between the sinusoidal pressure-waves generated by ultrasound transmitters and the random shock pressure waves generated by imploding bubbles $[38,57,58]$.

In order to fulfill the aim of the paper, we chose to adopt a modeling approach whereby the dynamics of bubble was investigated through numerical integration of the Keller-Miksis equations [40]. The pressure of the fluid hosting the bubble (i.e., the pressure forcing) was assumed to undergo stochastic fluctuations which were simulated using the Ornstein-Uhlenbeck model [59]. This model is well-established and represents a wide number of random processes in nature [60-63]. More importantly, it is characterized by only two free parameters that allow for a systematic exploration of noise-intensity and noise-autocorrelation effects on bubble dynamics.

\section{METHODS}

\section{A. Mathematical modeling of bubble dynamics}

We focus on a single bubble located far from solid boundaries or liquid surfaces. The bubble is assumed to be spherical, positionally stable, and its surface not to be affected by instability mechanisms [64]. In order to study the dynamics of this type of cavity, it is standard to focus on the temporal evolution of the bubble radius, $R(t)$, where $t$ is time. A well-known mathematical framework for the modeling of $R(t)$ is given by the Keller-Miksis equation [40]

$$
\left(1-\frac{\dot{R}}{c}\right) R \ddot{R}+\left(1-\frac{\dot{R}}{3 c}\right) \frac{3}{2} \dot{R}^{2}=\left(1+\frac{\dot{R}}{c}\right)\left(\frac{p_{w}-p(t)}{\rho}\right)+\frac{R}{c} \frac{\mathrm{d}}{\mathrm{d} t}\left(\frac{p_{w}-p(t)}{\rho}\right)
$$

where dots denote time derivation, $c$ is the speed of ${ }_{144}$ sound, $\rho$ is the liquid density, $p(t)$ is the (possibly time ${ }_{145}$ dependent) liquid pressure indefinitely far from the bub-146 ble wall (often indicated in the literature also as $p_{\infty}$ ), and ${ }_{147}$ $p_{w}$ is the liquid pressure at the bubble wall. We chose 148 the Keller-Miksis equation in place of more simplified for-149 mulations (e.g., the Rayleigh-Plesset equation) in order 150 to properly model large and fast temporal variations of ${ }_{151}$ the radius $R(t)$ [40]. In the following, we will show that 152 long-lasting and large increments of the bubbles' radius153 play a key role in determining chaos in the radius dynam-154 ics. In this regard, Nazari-Mahroo et al. [69] compared ${ }_{155}$ the Keller-Miksis, Gilmore, and Lezzi-Prosperetti models, and showed that - during the radius expansion stage - they behave very similarly. This means that the results presented herein are robust and overall insensitive to the choice of the specific bubbles' dynamics model. It should ${ }_{157}^{156}$ also be noted that during radius expansion stage, the bubble remains spherical. This is confirmed for instance by the experiments reported by Löfstedt et al. [70].

The bubble is assumed to contain a mixture of liquid vapor and non-condensible gas and to be submerged within a liquid at constant temperature. If this mixture behaves as an ideal gas, the total pressure inside the bubble can be evaluated as $p_{G}+p_{v}$, where $p_{G}$ and $p_{v}$ are the gas and vapor partial pressure inside the cavity, respectively. Under this assumption, the pressure at the bubble wall, $p_{w}$, can be derived by a force balance at the gas-liquid interface, reading

$$
p_{w}=p_{G}+p_{v}-\frac{2 S}{R}+4 \mu \frac{\dot{R}}{R},
$$

where $S$ is the surface tension, and $\mu$ is the liquid dynamic viscosity. Provided that the liquid that hosts the 
TABLE I. Physical parameters adopted for the liquid hosting ${ }^{190}$ the bubble. Data refer to water at $293 \mathrm{~K}$.

\begin{tabular}{ccccc}
\hline$\rho$ & $S$ & $\mu$ & $c$ & $p_{v}$ \\
$\left(\mathrm{~kg} \cdot \mathrm{m}^{-3}\right)$ & $\left(\mathrm{N} \cdot \mathrm{m}^{-1}\right)$ & $(\mathrm{Pa} \cdot \mathrm{s})$ & $\left(\mathrm{m} \cdot \mathrm{s}^{-1}\right)$ & $(\mathrm{Pa})$ \\
998 & $73 \cdot 10^{-3}$ & $1.00 \cdot 10^{-3}$ & 1481 & 2338 \\
\hline
\end{tabular}

bubble is kept at constant temperature, the vapor pres-197 sure inside the cavity, $p_{v}$, is also constant. The gas pres-198 sure inside the bubble, instead, can be evaluated accord-199 ing to the polytropic relationship

$$
p_{G}=p_{G, e q}\left(\frac{R_{e q}}{R}\right)^{3 k},
$$

where $p_{G, e q}$ and $R_{e q}$ are the gas pressure inside a bubble and the bubble radius in equilibrium conditions, respectively, and $k$ is the so-called polytropic exponent. In this study, bubbles are supposed to undergo adiabatic volume changes, i.e., $k=1.4$. This is consistent with several ${ }^{205}$ studies $[3,65,66]$ that have shown that bubble dynam-206 ics - as predicted by adiabatic mathematical models -207 matches experimental observations. Finally, the pressure ${ }^{208}$ inside a bubble in steady conditions, $p_{G, e q}$, is evaluated ${ }^{209}$ from (1) and (2) setting $\dot{R}=\ddot{R}=0$ as

$$
p_{G, e q}=p-p_{v}+\frac{2 S}{R_{e q}},
$$
where $p$ is the pressure of the liquid far from the bubble. ${ }_{213}^{212}$

A key parameter is the period of bubble free oscilla- ${ }_{214}$ tions $[3,25]$

$$
T_{n}=2 \pi\left(\frac{3 k\left(p-p_{v}\right)}{\rho R_{e q}^{2}}+\frac{2(3 k-1) S}{\rho R_{e q}^{3}}\right)^{-\frac{1}{2}} .
$$

This parameter will be crucial in the interpretation of the 220 temporal evolution of the cavity radius $R(t)$. In the fol-221 lowing, water at $293 \mathrm{~K}$ is assumed as hosting liquid, and 222 Table I reports the corresponding physical parameters. 223

The pressure of the liquid hosting the bubble is sup- ${ }^{228}$ posed to evolve over time as

$$
p(t)=\bar{p}+p^{\prime}(t)
$$
where $\bar{p}$ is the mean pressure experienced by the cav-233 ity, and $p^{\prime}(t)$ is the time-dependent fluctuation around ${ }_{234}$ $\bar{p}$. The fluctuations $p^{\prime}(t)$ are modeled as an Ornstein-235 Uhlenbeck process [59, 63].

The Ornstein-Uhlenbeck process is a stationary col-237 ored Gaussian-Markov process with the following char-238 acteristics: (i) the probability density function of the 239 realizations $p^{\prime}(t)$ is a normal distribution with zerOz40 mean and standard deviation $\sigma_{p}$; (ii) the stochastic process is exponentially autocorrelated as $\overline{p^{\prime}(t) p^{\prime}(t+\tau)}=$ $\sigma_{p} \exp \left[-\tau / \tau_{p}\right]$, where $\tau_{p}$ is the autocorrelation time-scale; and (iii) the process is stationary, namely $\sigma_{p}$ and $\tau_{p}$ do not change over time.

We have chosen the Ornstein-Uhlenbeck process as the random pressure forcing due to its simplicity, mathematical tractability and the possibility of changing its variance and (linear) memory by acting on only two parameters, namely the standard deviation $\sigma_{p}$ and the autocorrelation time-scale $\tau_{p}$.

From a numerical point of view, the realizations of the pressure fluctuations, $p^{\prime}(t)$, are evaluated by the socalled "exact update formula" provided by Gillespie [67], namely

$$
p^{\prime}(t+\Delta t)=p^{\prime}(t) \cdot \zeta+\sigma_{p} \cdot \sqrt{1-\zeta^{2}} \cdot n,
$$

where $n$ is a unit normal random number, $\Delta t$ is the time-step of the process and $\zeta=\exp \left[-\Delta t / \tau_{p}\right]$. Since (7) provides an exact update for $p^{\prime}(t)$, the actual value of the time-step of the process is arbitrary, and $\Delta t=\tau_{p} / 50$ was chosen in this study.

\section{Simulation of bubble radius dynamics}

In order to investigate the effect of the stochastic pressure forcing on the dynamics of a bubble (i.e., on the time-series of the bubble radius $R(t)$ ), a number of numerical simulations was performed. Each numerical simulation consisted of two steps. Firstly, a random pressure forcing $p(t)$ was simulated according to (7). Secondly, Equation (1) was forced with $p(t)$ and numerically solved to obtain the response of the bubble, namely the time-series of the radius $R(t)$.

Simulations of $p(\mathrm{t})$ were performed setting $\bar{p}=100 \cdot 10^{3}$ Pa. Three correlation times $\tau_{p}=[0.5,1,2] T_{n}$ were considered, and the standard deviation of the pressure was changed in the range $[0,120] \cdot 10^{3} \mathrm{~Pa}$. The duration of the simulations was set equal to $4000 T_{n}$. This duration guaranteed a robust estimation of all the statistical properties of $R(t)$, for all the investigated conditions.

In order to obtain $R(t)$ from the numerical integration of (1) with the forcing (7), the initial conditions $R(0)=$ $R_{e q}=5 \cdot 10^{-6} \mathrm{~m}$ and $\dot{R}(0)=\ddot{R}(0)=0$ were imposed and the time step $\Delta t=10^{-8} \mathrm{~s}$ was adopted. $R(t)$ was normalized with the equilibrium radius $R_{e q}[25,40,45]$ to better quantify the dynamics of the bubble radius.

Fig. $1 b$, reports the time-series of the normalized radius $R(t) / R_{e q}$ as obtained from integration of Eq. 1 when forced with the pressure reported in Fig. 1a. In Fig. $1 c-d$ the pdfs of the time-series $p(t)$ and $R(t)$ (partially reported in Panels $a-b$ ) illustrate the variability of $p(t)$ and $R(t)$. Similarly, Figs. $1 e-f$ report the autocorrelation functions, and illustrate how the correlation time is evaluated. 

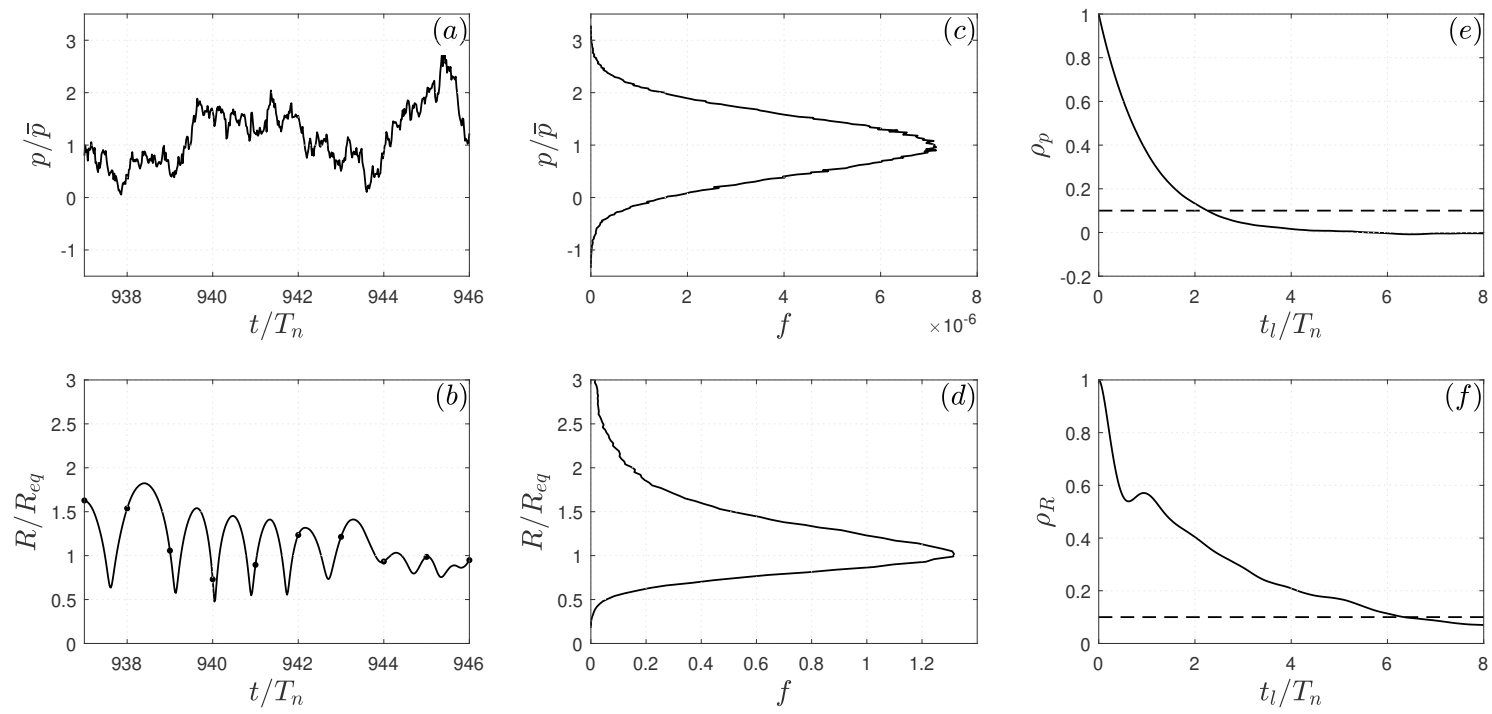

FIG. 1. (a) Example of a time-series of the normalized stochastic pressure forcing $p(t) / \bar{p}$. $(b)$ Time-series of the normalized radius $R(t) / R_{e q}$ of the bubble forced by the pressure reported in $(a)$. The black dots in $(b)$ highlight the bubble radius attained at the instants $n T_{n}$, where $n$ is an integer and $T_{n}$ is the natural oscillation period of the bubble (see Section III A for the explanation). $(c, d)$ Probability density functions and $(e, f)$ autocorrelation functions of the time-series (partially) reported in $(a, b)$. The dashed lines in $(e, f)$ mark the level $\rho_{p}=\rho_{R}=0.1$. It should be noted that the time-lag $\hat{t}_{l}$ such that $\rho\left(\hat{t}_{l}\right)=0.1$ is defined as the correlation time of the time-series. The times in $(a, b)$ and the time lags reported in $(e, f)$ are normalized by $T_{n}$. The adopted parameters are $\sigma_{p}=60 \cdot 10^{3} \mathrm{~Pa}$ and $\tau_{p}=2.0 T_{n}=2.8 \cdot 10^{-6} \mathrm{~s}$.

The interested reader can find in Appendix C further 266 details about the numerical techniques adopted to solve267 (1) and a sensitivity analysis of the solution with respect to: the time-step adopted for the numerical solution; the duration of the simulations; and the number of realiza ${ }_{269}{ }^{268}$ tions adopted for the statistical analyzes.

\section{RESULTS}

Four complementary perspectives are adopted to study ${ }^{277}$ the behavior of $R(t) / R_{e q}$. The first (Sec. A) is based on ${ }_{279}^{278}$ bifurcation diagrams and presents a way to identify the ${ }_{280}^{279}$ onset of chaos in the $R(t) / R_{e q}$ time-series. The second ${ }_{281}^{280}$ (Sec. B) investigates the physical mechanisms underpin- ${ }_{282}$ ning the onset of chaotic fluctuations. The third $\left(\operatorname{Sec}_{283}{ }_{282}\right.$ C) is a detailed statistical analysis of $R(t) / R_{e q}$, with ${ }_{284}^{283}$ a particular emphasis on the dependence of $R(t) / R_{e q_{285}}{ }^{284}$ statistical-moments on various combinations of noise in- ${ }_{286}$ tensity and correlation time scales. Finally, Sec. D digs ${ }^{286}$ deeper into second order statistics and investigates dom- ${ }^{287}$ inant modes and characteristic time scales of $R(t) / R_{e q}{ }^{288}$ time-series. This provides hints about the random $\mathrm{vs}^{289}$ organized temporal structure of $R(t)$.

All the results are wrapped up in Sec. IV, which pro-292 vides an overview of bubbles' behavior under stochastic 293 pressure forcing, using and harmonizing all the results294 obtained from Sec. III A, B, C and D.

\section{A. Assessment of the temporal pattern and bifurcation diagram}

We begin the results section by discussing the temporal dynamics exhibited by $R(t) / R_{e q}$. To this aim, the values $R\left(t=n T_{n}\right) / R_{e q}$ with $n=1,2, \ldots$ were extracted from $R(t) / R_{e q}$ (see dot-symbols in Fig. $1 b$ ). If the bubble radius oscillation exhibits a period $T_{n}, R(t)$ takes the same value at instants that are multiples of $T_{n}$. Conversely, if $R(t)$ is not periodic (or when the period of oscillations is different from $T_{n}$ ) then $R\left(n T_{n}\right) / R_{e q}$ exhibits a variability.

Figs. $2 a, b$ show results associated with the analysis of $R\left(n T_{n}\right) / R_{e q}$ in the form of noise-intensity bifurcation diagrams. These graphs report on the $\mathrm{x}$-axis the noise intensity $\sigma_{p} / \bar{p}$ and on the y-axis the values of $R\left(n T_{n}\right) / R_{e q}$ extracted from the corresponding time-series $R(t)$. The gray and red dots in Panels $2 a$ and $2 b$ refer to different correlation times $\tau_{p}$. The noise-intensity-bifurcation diagrams obtained in Figs. $2 a, b$ align with those obtained from other studies that considered a sinusoidal forcing $[25,40,45]$, but key differences can be observed.

In the case of a sinusoidal forcing with amplitude $A_{p}$ and period $T_{n}$, the noise-intensity-bifurcation diagrams exhibit two different zones. When $A_{p}$ is lower than a threshold $A_{p, c}$, the metric $R\left(n T_{n}\right) / R_{e q}$ is perfectly constant for any $n$. This can be seen, for example, in Figs. $3 a, b$ that report the radius dynamics forced by the sinusoidal pressure with amplitude $A_{p}<A_{p, c}$ shown in Figs. $3 d$,e. Differently, for $A_{p}>A_{p, c}$ the metric $R\left(n T_{n}\right) / R_{e q}$ exhibits a large variability for a fixed value of $A_{p}$ and for 

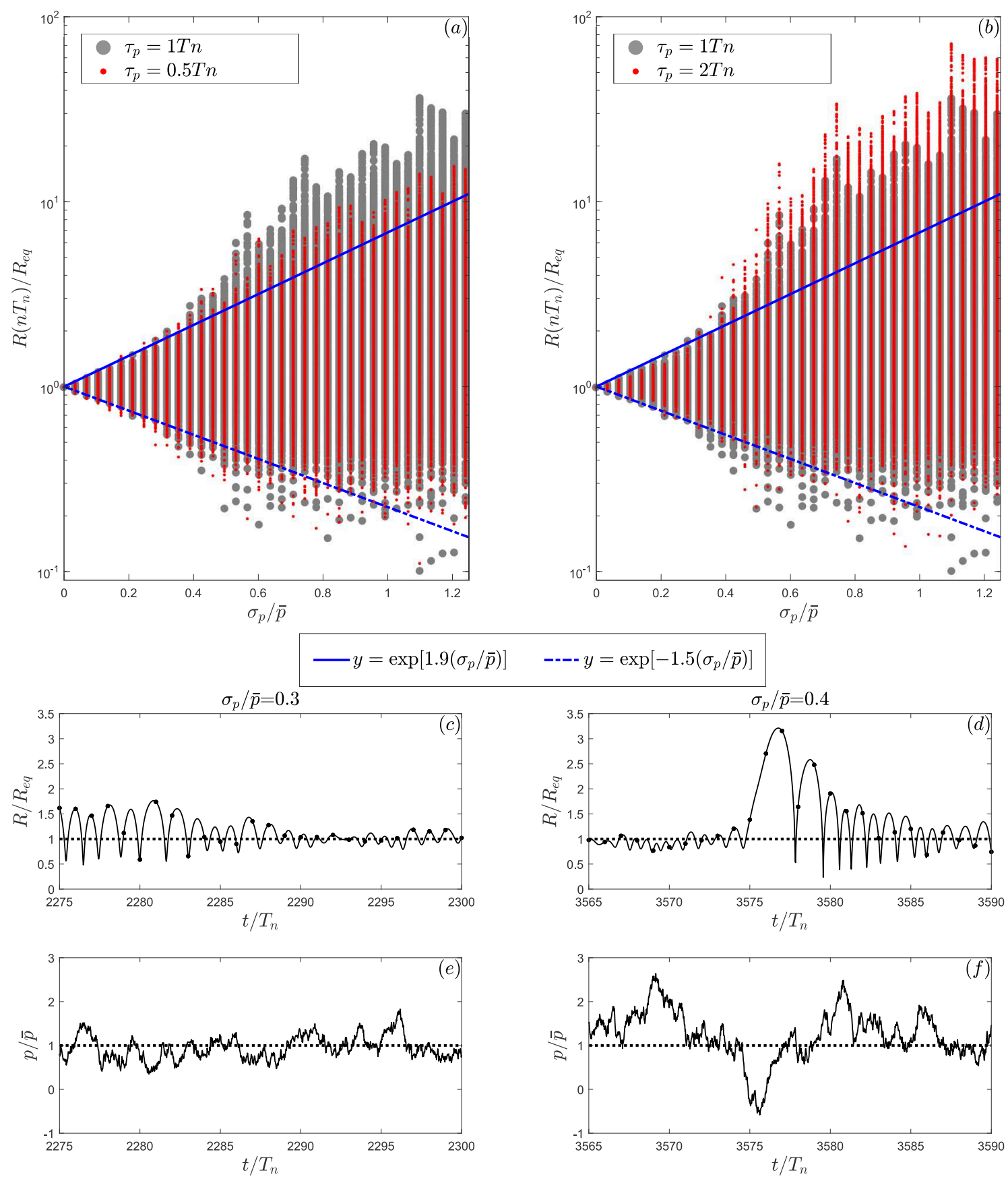

FIG. 2. ( $(a, b)$ Noise-intensity bifurcation diagrams. For a given value of $\sigma_{p} / \bar{p}$, the dynamics of $R(t)$ is simulated for $4000 T_{n}$. From this simulation, only the values $R\left(n T_{n}\right) / R_{e q}$ are selected, and are reported in the vertical axis for the given $\sigma_{p} / \bar{p}$. In both Panels, the gray circles refer to $\tau_{p}=T_{n}$. In Panel $(a)$ and $(b)$ the red dots refer to $\tau_{p}=T_{n} / 2$ and $\tau_{p}=2 T_{n}$, respectively. $(c-f)$ Time segments of the time-series $R(t) / R_{e q}$ and $p(t) / \bar{p}$. The horizontal dotted lines mark the equilibrium radius $R(t) / R_{e q}=1$ and the mean pressure $p(t) / \bar{p}=1$. The black dots in $(c-d)$ highlight the bubble radius attained at the instants $n T_{n}$. Panels $(c, e)$ refer to $\sigma_{p} / \bar{p}=0.3$ and Panels $(d, f)$ refer to $\sigma_{p} / \bar{p}=0.4$; in both cases, $\tau_{p}=T_{n}$.

different values of $n$. This non-regular behavior is exem-302 plified in Fig. $3 c$, which shows the radius dynamics under 303 the sinusoidal pressure forcing with amplitude $A_{p}>A_{p, c} 304$ of Fig. $3 f$. Therefore, in the case of a sinusoidal forc-305 ing, $A_{p, c}$ represents an amplitude threshold that sharply 306 separates the non chaotic and chaotic regimes.
When stochastic fluctuations of pressure are considered, the variability of $R\left(n T_{n}\right) / R_{e q}$ increases with increasing $\sigma_{p} / \bar{p}$ (Figs. 2a,b). This is consistent with the case of a sinusoidal forcing. However, while $\sigma_{p} / \bar{p}$ increases, a clear threshold that separates regular oscillations from chaotic fluctuations does not emerge. 

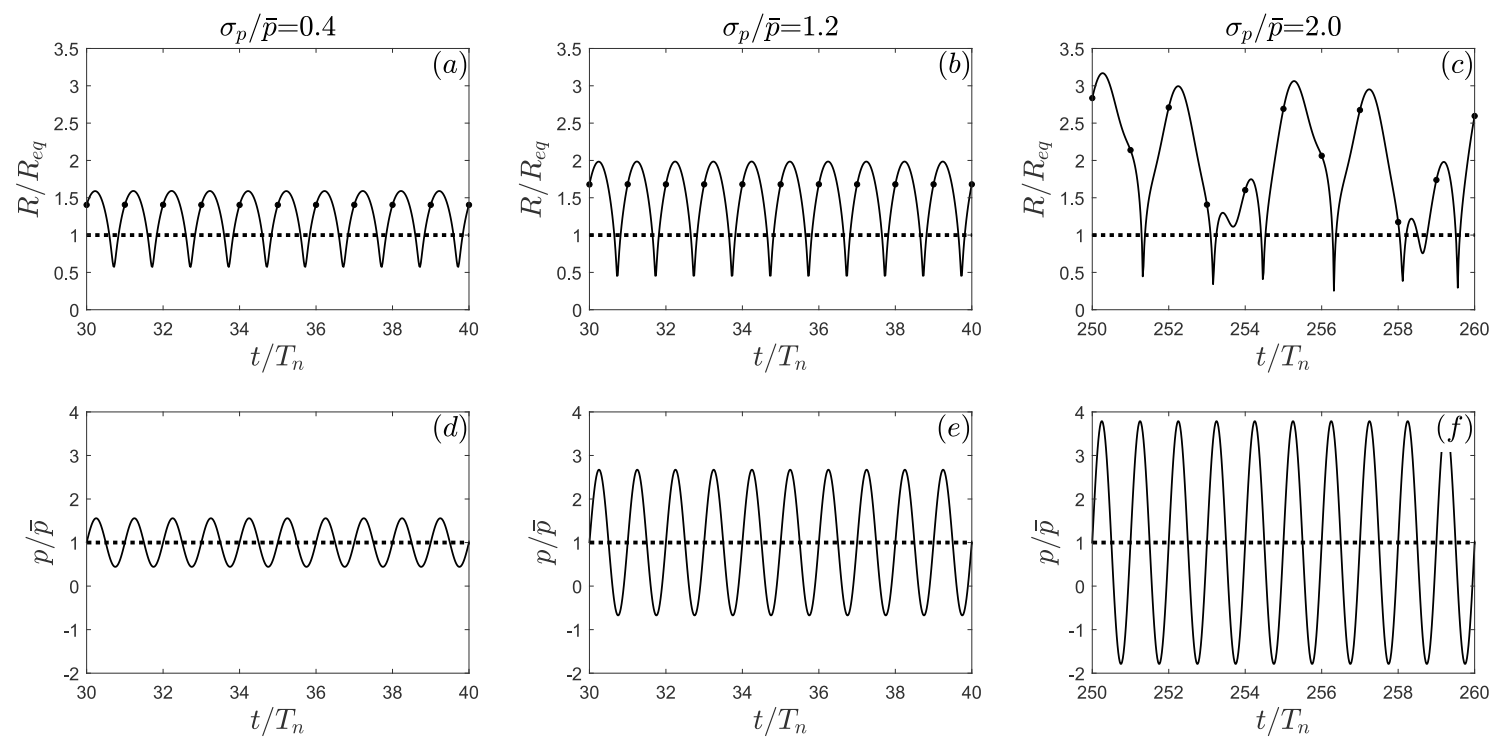

FIG. 3. Time segments of the time-series $R(t) / R_{e q}$ (top row) and $p(t) / \bar{p}$ (bottom row) when a sinusoidal external pressure with amplitude $A_{p}=\sqrt{2} \sigma_{p}$ and period $\tau_{p}=T_{n}$ - is applied. The dotted lines mark the equilibrium radius $R(t) / R_{e q}=1$ and the mean pressure $p(t) / \bar{p}=1$. The black dots in $(a-c)$ highlight the bubble radius attained at the instants $n T_{n}$. Panels $(a, d)$, $(b, e)$ and $(c, f)$ refer to $\sigma_{p} / \bar{p}=0.4, \sigma_{p} / \bar{p}=1.2$ and $\sigma_{p} / \bar{p}=2.0$, respectively.

Moreover, even for very low values of $\sigma_{p} / \bar{p}$, the metric338 $R\left(n T_{n}\right) / R_{e q}$ does show some level of variability and hence $3 з 9$ it is not constant.

A more careful inspection shows that a change in the341 bubble dynamics occurs at $\sigma_{p} / \bar{p} \approx 0.30$ : for $\sigma_{p} / \bar{p} \lesssim 0.30,342$ the normalized radius oscillates around 1 and is con-343 fined by the almost symmetrical curves $\exp \left[1.9\left(\sigma_{p} / \bar{p}\right)\right]_{344}$ and $\exp \left[-1.5\left(\sigma_{p} / \bar{p}\right)\right]$ (these curves were obtained by fit-345 ting the maximum and minimum values attained by 346 $R\left(n T_{n}\right) / R_{e q}$ for $\left.\sigma_{p} / \bar{p}<0.30\right)$; differently, for $\sigma_{p} / \bar{p} \gtrsim 0.30,347$ the variability of the radius suddenly increases and 348 $R\left(n T_{n}\right) / R_{e q} \in[0.01,50]$.

\section{B. Physics of chaos inception}

In order to elucidate the physical behavior behind the 354 inception of chaos in the dynamics of $R(t)$ occurring for ${ }_{355}$ $\sigma_{p} / \bar{p}>0.3$, Panels $2 c-d$ report two exemplifying por-356 tions of time-series $R(t) / R_{e q}$. To relate the bubble radius ${ }_{357}$ dynamics to the pressure fluctuations, the corresponding ${ }_{358}$ time-series $p(t) / \bar{p}$ are reported in Panels $2 e-f$. These 359 pressure time-series are obtained setting the same noise $e_{300}$ time-scale $\tau_{p}=T_{n}$ but different noise intensities. The $\mathrm{e}_{361}$ dotted lines mark the threshold $p(t) / \bar{p}=1$, and help to ${ }_{362}$ discern the instants when the instantaneous forcing pres-363 sure is below average (i.e., $p(t) / \bar{p}<1$ ) or above average ent $_{64}$ (i.e., $p(t) / \bar{p}>1$ ). We recall that when the instantaneous ${ }_{365}$ pressure is below/above average, the bubble radius tends ${ }_{366}$ to increase/decrease.

Panels $2 c, e$ refer to the noise intensity $\sigma_{p} / \bar{p}=0.3368$ (i.e., just below the threshold that separates the non-369 chaotic/chaotic behaviors). In this case, the pressure os- з70 cillates slightly around the mean value (Panel 2e) and the bubble radius does not undergo large increments $\left(R(t) / R_{e q}\right.$ never exceeds the value 2 , see Panel $\left.2 c\right)$. It follows that during the small radius increments little energy is stored in the bubble. As a consequence of this: (i) the subsequent rebound is mild $\left(R(t) / R_{e q}\right.$ remains close to unity); and (ii) the radius growth that follows the rebound is mild as well. The radius dynamics is therefore characterized by a sequence of modest increments of radius intercut with mild rebounds. At this conditions, the period of the oscillations is very close to the natural oscillation period of the bubble and no chaos is detected.

In contrast, Panels $2 d, f$ focus on the noise intensity $\sigma_{p} / \bar{p}=0.4$ (i.e., above the no-chaos/chaos threshold). In this case, the pressure may deviate significantly from the mean value (e.g., see immediately after $t / T_{n}=3575$ in Panel 2f). As a result, large increments in the bubble radius occur, that may last a few times the natural period $T_{n}$. For instance, this can be seen in Panel $2 d$, where the radius growth starting at $t / T_{n} \approx 3575$ lasts about $3 T_{n}$, and $R(t) / R_{e q}$ eventually exceeds the value 3. During these large increments of radius, a significant amount of energy is stored in the bubble. Consequently: (i) the subsequent rebound is violent $\left(R(t) / R_{e q}\right.$ is much lower than unity); and (ii) the radius growth that follows the rebound may be considerable and long lasting (this is exemplified in in Panel $2 d$, where the radius growth that begins after the rebound at $t / T_{n} \approx 3578$ lasts about $2 T_{n}$ ). The radius dynamics is therefore characterized by a sequence of significant and long lasting increments of radius (the duration of these phases exhibit a wide variability) intercut with violent rebounds. At these conditions, $R(t) / R_{e q}$ deviates significantly from unity, and 
the period of the oscillations varies significantly from the 429 natural oscillation period of the bubble. Accordingly, a430 chaotic behavior is detected. It should be noted that the ${ }_{431}$ behaviors reported in the time segments of Panels $2 c-f_{432}$ are not rare, but are detected in a large number of time ${ }_{433}$ segments in the time-series simulated in this work.

The examples previously reported depict a picture435 where bubble chaotic dynamics is characterized by long ${ }^{436}$ lasting and large radius increments, induced by time-437 coherent negative pressure fluctuations. It follows that438 chaos occurs when downcrossing events in the pressure 439 signal exceed suitable thresholds; namely, the duration440 and the magnitude of the negative pressure fluctuations441 (with respect to the pressure mean value) become suffi-442 ciently high. In the cases investigated in this work, such ${ }_{443}$ downcrossing analysis gives that bubble chaotic dynam-444 ics occurs when: (i) the duration of pressure reduction445 events exceeds the threshold $1.5 T_{n}$; and (ii) the corre-446 sponding mean value of the pressure reduction during ${ }_{447}$ this negative pressure events is greater than $0.6 \bar{p}$. How-448 ever, it should be noted that the bubble response to pres-449 sure forcing depends on the physical properties of fluid450 and the initial size of the bubble. Therefore, the physics451 of chaos inception previously described (i.e., interplay 452 between long lasting, intense pressure fluctuations and453 nonlinear bubble dynamics) is of general validity. How-454 ever, the exact threshold values dictating the transition455 to chaos detected here are are surely dependent on the fluid characteristics (see Table I). The precise determination of this dependence is beyond the scope of the present ${ }_{456}$ work, and will be the subject of future work.

We now briefly highlight the key role of pressure ${ }_{457}$ stochasticity in the inception of chaos in bubbles' dy- $_{458}$ namics. To this aim, we evaluated the response of ${ }_{459}$ a bubble to three sinusoidal pressure forcing $p(t) / \bar{p}={ }_{460}$ $1+\left(A_{p} / \bar{p}\right) \sin \left(2 \pi t / T_{n}\right)$, and compared it against the be- ${ }_{461}$ havior depicted in Panels $2 d, f$. Three relevant val- ${ }_{462}$ ues of the oscillation amplitude, $A_{p}$, were tested: $(\mathrm{i})_{463}^{462}$ $A_{p} / \bar{p}=\sqrt{2} \cdot 0.4$, such that the standard deviation of ${ }_{464}$ the sinusoidal signal is $\sigma_{p}=0.4 \cdot \bar{p}$, and the resulting ${ }_{465}$ radius dynamics can be compared with Panel $2 d$ (that $_{466}$ refers to a stochastic pressure forcing with $\left.\sigma_{p} / \bar{p}=0.4\right)_{; 467}$ (ii) $A_{p} / \bar{p}=\sqrt{2} \cdot 1.2$, (i.e., the sinusoidal forcing is char-468 acterized by $\sigma_{p} / \bar{p}=1.2$ ) such that the minimum pres-469 sure attained by the sinusoidal forcing is the same typ- 470 ically attained by the stochastic forcing of Panel $2 f ; ;_{471}$ and (iii) $A_{p} / \bar{p}=\sqrt{2} \cdot 2.0$, inducing pressure oscillations472 with $\sigma_{p} / \bar{p}=2.0$, i.e., much higher than 0.4. Results 473 on $R(t) / R_{e q}$ are reported in Fig. 3. The noise intensities 474 $\sigma_{p} / \bar{p}=0.4$ and $\sigma_{p} / \bar{p}=1.2$ (Panels $3 d-e$ ) did not lead to $0_{475}$ inception of chaos: the radius time-series were very reg-476 ular and exhibited fluctuations with the constant period ${ }_{477}$ $T_{n}$ (Figs. $\left.3 a-b\right)$. Differently, for $\sigma_{p} / \bar{p}=2.0(\text { Panel } 3 f)_{\text {,478 }}$ a chaotic behavior of the bubble radius occurred ( Panel $_{479}$ $3 c$ ).

The comparison of results shown in Fig. 2 (related to481 random forcing) and in Fig. 3 (corresponding to sinu-482 soidal forcing) clearly shows that stochasticity promotes483 the chaos inception. Although sinusoidal pressure signals have the same standard deviation $\left(\sigma_{p} / \bar{p}=0.4\right.$, Panels $3 a, d)$ or the same typical minimum values (Panel $3 b, e$ ) of the stochastic forcing, sinusoidal pressure forcing do not lead to chaotic bubble dynamics, while random forcing does. Only the increment of the oscillation amplitude of the sinusoidal pressure to $A_{p} / \bar{p}=\sqrt{2} \cdot 2.0$ eventually lead to the inception of chaos. Namely, the noise intensity of the sinusoidal pressure should be five times larger than that of the stochastic case, in order to observe a similar pattern of chaotic radius fluctuations.

The role of the correlation time of the forcing, $\tau_{p}$, was also explored. Red dots in Figs. 2a,b correspond to $\tau_{p}=0.5 T_{n}$ and $\tau_{p}=2 T_{n}$, respectively; in each panel data pertaining to $\tau_{p}=T_{n}$ (gray circles in both panels) are kept to allow for comparisons. It emerges that variations of $\tau_{p}$ are relevant only for $\sigma_{p} / \bar{p} \gtrsim 0.30$ (i.e., above the threshold identified before) and positively correlated with the variability of the bubble radius. This behavior is in accordance with the physical explanation of the inception of chaos described so far. Higher values of correlation time of the forcing entail longer periods over which the pressure fluctuation has a constant sign. Hence, longer periods of pressure below average can be observed. These, in turn, promote large radius increments and thus the inception of chaos. This analysis is performed in more details in the Appendix A.

\section{Statistical analysis}

The analysis of Fig. 2 reveals that $R(t)$ deviates significantly from its equilibrium value and the behavior of $R(t)$ can be very irregular. In order to better quantify the deviations of $R(t)$ from $R_{e q}$, the probability density functions (pdf) and the cumulative distribution functions (cdf) of the metric $R / R_{e q}$ were evaluated. Details about this statistical analysis are given in the Appendix B where we report that changes in both $\sigma_{p} / \bar{p}$ and $\tau_{p}$ induce significant alterations in the pdf of the bubble radius $R(t)$. However, $\sigma_{p} / \bar{p}-$ effects seems to be stronger. For this reason, the effect of $\sigma_{p} / \bar{p}$ was systematically explored in the relatively large range $[0,1.20]$ for only three values of the noise correlation time $\tau_{p}=[0.5,1,2] T_{n}$.

For the sake of clarity, the corresponding effects on the pdfs of $R(t)$ are then expressed in terms of four relevant statistical parameters, reported in Fig. 4: (i) the mean value of the normalized bubble radius, $\bar{R} / R_{e q}$; (ii) the coefficient of variation of $R(t)$, i.e., $c_{V, R}=\sigma_{R} / \bar{R}$; (iii) the skewness $s_{R}$ of the time-series; and (iv) the kurtosis $k_{R}$ of $R(t)$.

The noise intensity $\sigma_{p} / \bar{p}$ has a strong effect on the mean value of the bubble radius (Fig. 4a). In particular, $\sigma_{p} / \bar{p}$ is positively correlated with $\bar{R}$. This is a key point: the mean value of the bubble radius depends not only on the mean pressure, $\bar{p}$, but also on the noise intensity, $\sigma_{p}$. Therefore, in the case of a stochastic pressure forcing, it can be misleading to estimate the mean value of the 

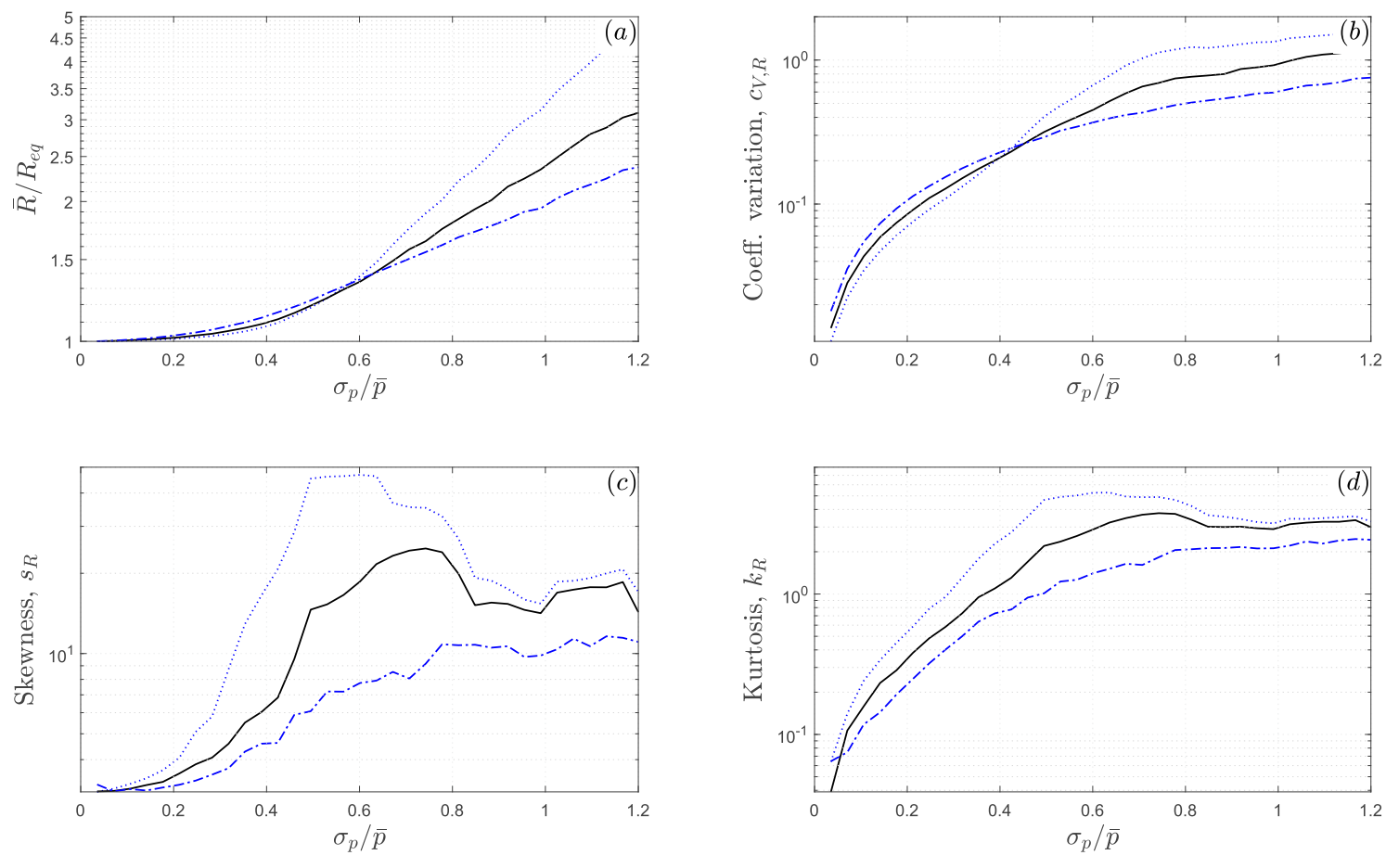

$-\cdots \tau_{p}=0.5 T_{n} \quad-\quad \tau_{p}=T_{n} \quad \cdots \cdots \cdot \tau_{p}=2 T_{n}$

FIG. 4. Effect of $\sigma_{p} / \bar{p}$ on some relevant statistical parameters that describe the time-series $R(t)$.

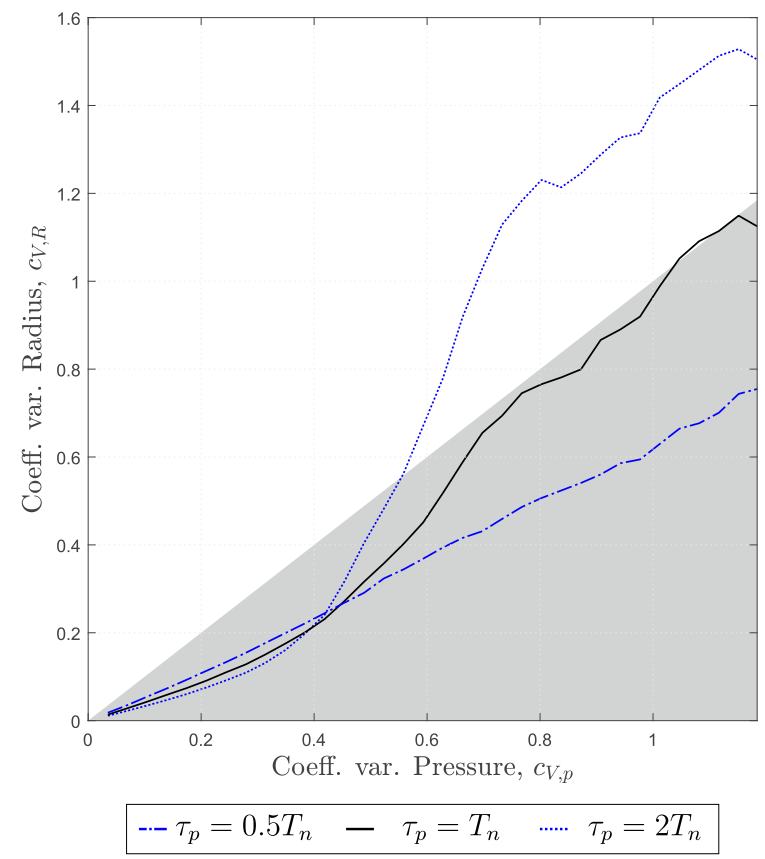

FIG. 5. Effect of the coefficient of variation of the pressure, ${ }^{505}$ $c_{V, p}$, on the coefficient of variation of the bubble radius, $c_{V, R}{ }^{506}$ The shaded zone highlights the lower half plane bounded by ${ }^{507}$ the bisector, where bubble exhibits the "damper" behavior. ${ }^{508}$ In the upper half plane bubble behaves as "amplifier".

bubble radius just from the mean (background) pressure.

When $\sigma_{p} / \bar{p}$ exceeds 0.60 , different curves $\bar{R} / R_{e q}$ are observed for different values of $\tau_{p}$. This can be explained as follows. According to the analysis presented in Section III A, the deviation of $\bar{R}$ from $R_{e q}$ is due to the nonlinear nature of the bubble dynamics and, in particular, it is ascribable to the effect of time segments during which the instantaneous pressure is below average (i.e., when $p(t)<\bar{p})$. When the pressure is below average, the bubble radius undergoes a strong increment and deviates significantly from $R_{e q}$ (i.e., the equilibrium radius attained at $p(t)=\bar{p}$, see Panels $2 c, d)$. This, clearly, contributes to increase $\bar{R}$. It was also pointed out that, the higher $\tau_{p}$, the longer the duration of time segments during which the instantaneous pressure is below average (see the Appendix A), and thus the stronger the increments of the bubble radius and, consequently, of $\bar{R}$ from $R_{e q}$. Besides $\bar{R}$, the other statistical parameters are all also strongly affected by the noise intensity (see Panels $4 b-d$ ).

The correlation time $\tau_{p}$ does not change the qualitative behavior of the the curves presented in Fig. 4, however, some peculiarities do occur: (i) the effect of $\tau_{p}$ on the mean value and on the coefficient of variation of $R(t)$ is most relevant for high values of $\sigma_{p} / \bar{p}$ (Panels $4 a, b$ ); (ii) the skewness and the kurtosis are affected by $\tau_{p}$ the most when $\sigma_{p} / \bar{p}$ is in the range [0.4, 0.8] (see Panels $4 c, d$ ), instead the curves tend to merge for higher values of the correlation time of the pressure forcing.

The behavior of skewness and kurtosis shows other 

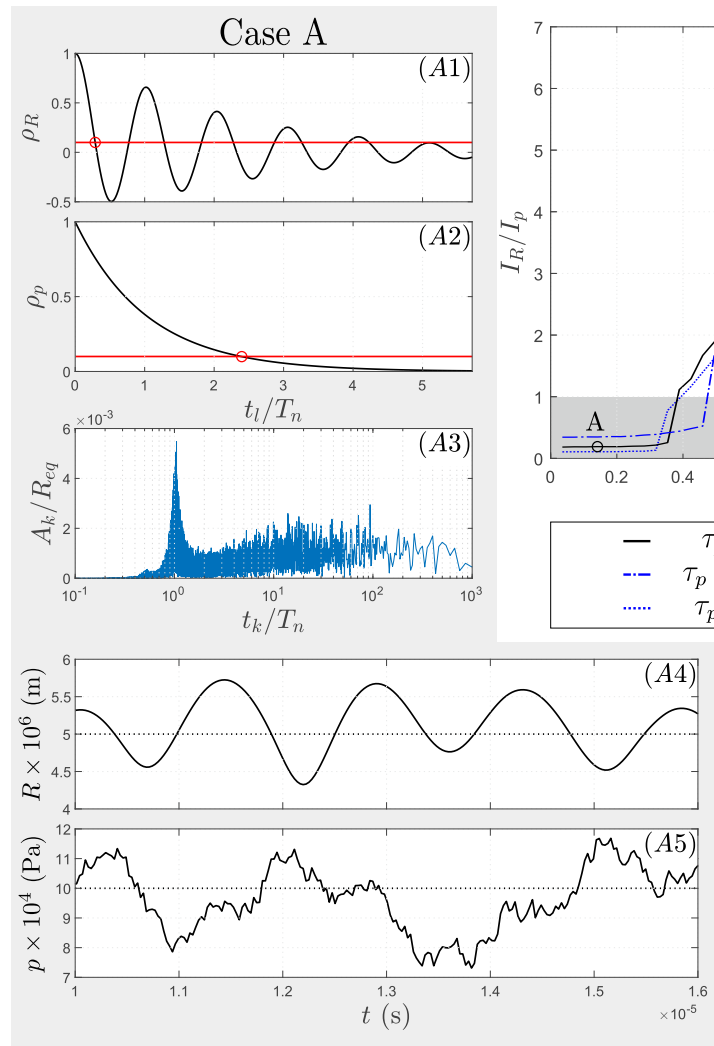
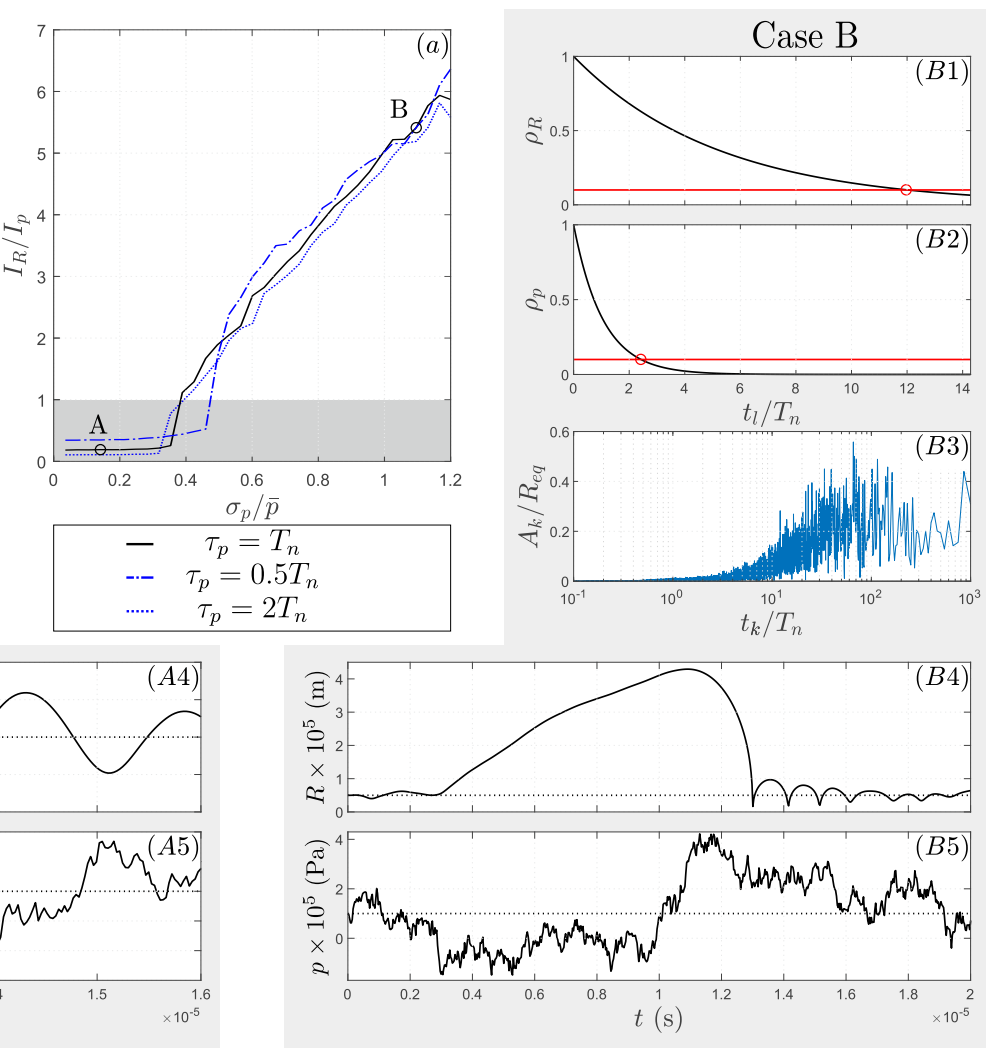

FIG. 6. (a) Effect of the noise intensity $\sigma_{p} / \bar{p}$ on the ratio between the integral scale of the radius time-series, $I_{R}$, and the integral scale of the pressure forcing, $I_{p}$. The gray zone highlights the condition $I_{R}<I_{p}$. Autocorrelation diagrams of $R(t)$ (Panels $A 1, B 1$ ) and $p(t)$ (Panels $A 2, B 2$ ). The red lines mark the level where the autocorrelation function is 0.1 . (A3, B3) Power (amplitude) spectrum of $R(t)$. It should be noted that the horizontal axis reports the period of the $k$-th harmonics (rather than its frequency). ( $A 4-B 5)$ Relevant time segment of the time-series $R(t)$ and $p(t)$. The dotted lines mark the equilibrium radius $R_{e q}$ and the mean pressure $\bar{p}$.

interesting aspects. For all investigated values of $\tau_{p}, 536$ they increase with increasing $\sigma_{p} / \bar{p}$ within the range 537 $\sigma_{p} / \bar{p}=[0,0.60]$. For $\sigma_{p} / \bar{p} \gtrsim 0.60$, instead, they seem538 to tend monotonically (kurtosis) or non-monotonically539 (skewness) to an asymptotic value (Panels $4 c, d$ ). In-540 terestingly, the kurtosis tends to its Gaussian value of $5_{541}$ three. In summary, the trends observed in Fig. 4 indicate ${ }_{542}$ that increments in the noise intensity tend to increase ${ }_{543}$ the mean radius of the bubble as well as the intensity544 of its variations (Panels $a$ and $b$ ). The positive value of $5_{545}$ the skewness indicates that it is more probable to have 546 $R(t)>R_{e q}$ than $R(t)<R_{e q}$. This asymmetry increases with increasing $\sigma_{p} / \bar{p}$ but saturates for $\sigma_{p} / \bar{p} \gtrsim 0.60$. The behavior depicted by kurtosis indicates that the occur- ${ }_{547}$ rence of extreme events (i.e., intermittency) in $R(t)$ increases with increasing noise intensity, but, as per the skewness, it saturates for $\sigma_{p} / \bar{p} \gtrsim 0.60$.

An important aspect in studies about nonlinear oscil- ${ }^{550}$ lators is to evaluate whether the system behaves as $\mathrm{a}^{551}$ "damper" or as an "amplifier" of the external forcing ${ }^{552}$ [54]. To this end, the variability of the bubble radius ${ }^{553}$ was compared to the variability of the forcing pressure 554 forcing (see Fig. 5). The gas bubble can be classified ${ }_{555}$ as a "damper" when the coefficient of variation of the fluctuating pressure forcing is larger than the coefficient of variation of the fluctuating bubble radius (i.e., $c_{V, p}>$ $c_{V, R}$, gray zone in Fig. 5). On the other end, if $c_{V, p}<$ $c_{V, R}$ (white zone in Fig. 5) the gas bubble behaves as a noise "amplifier". The correlation time of the noise, $\tau_{p}$ is a key parameter in determining the amplifier/damper behavior of the bubble oscillator. For $\tau_{p} \leq T_{n}$ the bubble dynamics usually exhibits a "damper" behavior. Differently, when $\tau_{p}=2 T_{n}$, the bubble behaves as a noise "amplifier" for $c_{V, p} \gtrsim 0.5$.

\section{Temporal correlation}

It is now instructive to analyze the correlation timescale of the radius signal $R(t)$. To this end, we evaluate the autocorrelation function $\rho_{R}\left(t_{l}\right)$ (see the examples reported in Panels $6 A 1, B 1)$. Then, we select the turnover time-lag $\hat{t}_{l, R}$ so that $\rho_{R}\left(\hat{t}_{l, R}\right)=0.1$ (red circles in Figs. $6 A 1, B 1)$. Finally, the integral scale of the signal is evaluated as $I_{R}=\int_{0}^{\hat{t}_{l, R}} \rho_{R}\left(t_{l}\right) \mathrm{d} t_{l}$. If the same procedure is applied to the time-series $p(t)$ (see Panels $6 A 2, B 2$ ), the 
integral scale of the noise $I_{p}=\tau_{p}$ is obtained. In order 614 to highlight the non-linear behavior of the bubble oscil-615 lator, we focus on the ratio between the integral scale of 616 the bubble radius and the integral scale of the pressure,617 namely $I_{R} / I_{p}$ (Fig. $6 a$ ). Note that the definition of the618 crossover time scale based on the $\rho_{R}=0.1$ is arbitrary.619 Note also, that any other value of $\rho_{R}$ reasonably close t0620 0 proved to lead to almost identical results and trends $s_{621}$ presented in Fig. 6a, meaning that the results discussed ${ }_{622}$ in what follows are essentially independent on the exact ${ }_{623}$ definition of the crossover time scale.

Fig. $6 a$ shows the effect of the noise intensity $\sigma_{p} / \bar{p}$ on625 $I_{R} / I_{p}$ and two contrasting behaviors are observed. When626 the noise intensity $\sigma_{p} / \bar{p}$ is lower or greater than $\approx 0.30627$ (this value depends slightly on $\tau_{p}$ ), then $I_{R} \ll I_{p}$ (gray628 zone in Fig. 6a) and $I_{R} \gg I_{p}$, (white zone in Fig. 6a),629 respectively.

In order to investigate the physical processes under-631 pinning this sharp change in the behavior of $I_{R} / I_{p}$, we $\mathrm{e}^{632}$ select two values of $\sigma_{p} / \bar{p}$ for which these contrasting be-633 haviors are observed (see points $\mathrm{A}$ and $\mathrm{B}$ in Fig. 6a). ${ }^{634}$ For both cases, the radius signal $R(t)$ (Figs. 6A4,B4) $)^{635}$ and the pressure signal $p(t)$ (Figs. $6 A 5, B 5$ ) are also re-636 ported over a significant time interval. Moreover, the ${ }^{637}$ power spectrum of $R(t)$ is evaluated (Figs. 6A3, B3). ${ }_{638}$

Case A. For low values of the noise intensity, the only ${ }^{639}$ effect of pressure fluctuations is to excite the free oscillations of the bubble. For instance, when $\sigma_{p} / \bar{p}=0.14$, the bubble radius oscillates with a varying amplitude (see ${ }^{640}$ Fig. 6A4), but the oscillation period is almost constant, and close to the natural period of oscillation of the bub-641 ble, $T_{n}$. This is confirmed by: (i) the peak in the power ${ }_{642}$ spectrum of $R(t)$ (Fig. 6A3); and (ii) the shape of the au-643 tocorrelation function (Fig. 6A1), which resembles that ${ }_{644}$ of a periodic signal with period equal to $T_{n}$. Therefore,645 for low noise intensity levels, pressure variations are not ${ }_{646}$ able to significantly alter the free oscillations of the bub-647 bles and induce chaos.

Case B. For high values of the noise intensity, pres-649 sure fluctuations drive the bubble dynamics. In the con-650 sidered case (the noise intensity is $\sigma_{p} / \bar{p}=1.10$ ), the651 bubble exhibits oscillations that attain large amplitudes652 (Fig. 6B4). Differently from Case A, the oscillation pe-653 riod undergoes strong variations in the range $[0.5,10] T_{n} .654$ As a result, the power spectrum of $R(t)$ (see Fig. 6B3) 655 does not show any clear peak, and harmonics with peri-656 ods in the wide range $\left[10^{1}, 10^{3}\right] T_{n}$ are characterized by657 comparable amplitudes. The signal portions reported in 658 Fig. $6 B 4, B 5$ show that pressure variations alter to a659 major extent the dynamics of the bubble - according660 to the physical mechanisms explained in Sec. III B -,661 and free oscillations with period $T_{n}$ are rarely observed.662 For instance, during the very long time segment from663 $t \approx 2 \cdot 10^{-6}$ s to $t \approx 10 \cdot 10^{-6} \mathrm{~s}$, the bubble radius be-664 comes very large $(\approx 10$ times the equilibrium value, see665 Fig. 6B4). After this long growth phase, oscillations with 666 a period slightly higher than $T_{n}$ are observed. The high 667 values of $I_{R}$ observed for high values $\sigma_{p}$ are therefore in-668 duced by the long periods over which a constant growth of $R(t)$ takes place. Note that, these long lasting growth phases are followed by rebounds exhibiting a period comparable to the bubble natural period. It follows that the increment of $I_{R}$ due to long lasting radius growth phases cannot be balanced by phases during which the bubble oscillates with a period close to $T_{n}$.

The behavior previously described justifies the negligible effect of noise correlation time on bubbles' dynamics observed when the noise intensity is below the nochaos/chaos threshold. This result was detected in the Fig. $2 a, b$ (see Sec. III B). When the noise intensity is below the no-chaos/chaos threshold, bubbles oscillate at their natural frequency, and the only role of pressure fluctuation is to provide energy to sustain this motion. The characteristics of such pressure fluctuations are irrelevant in determining the frequency of vibration of the bubble. At most, they slightly alter the amplitude of the radius oscillation. Differently, when the noise intensity is above the no-chaos/chaos threshold, the bubble's dynamics are strongly driven by the pressure forcing. Hence, key characteristics of the pressure fluctuation - such as the noise correlation time - become important in determining bubble dynamics. In particular, longer correlation times according to the mechanisms illustrated in Section III B - are associated with a more chaotic bubble response.

\section{CONCLUSIONS}

The response of a single bubble to a stochastic pressure forcing was investigated. The motivation underpinning this study lies: (i) in the occurrence of random pressure fluctuations in many applications exploiting bubble dynamics; and (ii) in the strong nonlinearities affecting the deterministic bubble dynamics, which suggests the possible occurrence of non-trivial noise-induced phenomena.

Two key parameters control stochastic bubble dynamics: the ratio between the standard deviation and the mean value of the forcing pressure $\left(\sigma_{p} / \bar{p}\right)$, and the ratio between the noise correlation time-scale and the period of bubble free oscillations $\left(\tau_{p} / T_{n}\right)$. Two typical behaviors were detected. The first one occurs when $\sigma_{p} / \bar{p}$ is lower than a threshold value around 0.3 ; namely, when pressure fluctuates with small amplitudes. In this case, the random pressure forcing mainly excites the free oscillations of the bubble whose radius undergoes small amplitude oscillations and exhibits a rather regular periodicity. Moreover, we observed that (i) the effect of $\tau_{p} / T_{n}$ is small, (ii) the mean value of the background pressure can be adopted to estimate the mean value of the bubble radius, and (iii) bubble always behaves as a damper of external noise.

The second behavior occurs when the fluid hosting the bubble experiences large-amplitude pressure fluctuations (i.e., $\sigma_{p} / \bar{p}>0.3$ ). At these conditions, pressure stochasticity is able to trigger a chaotic bubble dynamics. Timeseries of the bubble radius exhibit large amplitude fluc- 
tuations and no evident periodicities occur, not even at721 the bubble natural frequency. The parameter $\tau_{p} / T_{n}$ now 722 significantly affects the bubble dynamics. In particular,723 when $\tau_{p} / T_{n}$ is high, long time intervals during which the 224 instantaneous pressure is below the mean pressure ap-725 pear; these intervals entail large increments of $R(t)$ and 726 are usually followed by cavities' collapses and rebounds.727 A strong variability of the $R(t)$ time-series occurs and the 228 bubble behaves as a nonlinear oscillator that amplifiesz29 the external noise. Consequently, the mean value of the rzo background pressure cannot be adopted to estimate the ${ }_{731}$ mean value of the bubble radius; in doing so, the mean ra-732 dius of the bubble can be underestimated of a factor five.733 It should be finally remarked the key role of stochastic- 734 ity in triggering chaos in bubble's radius dynamics. Two 735 pressure forcing -one stochastic, one sinusoidal- char-736 acterized by the same noise intensity $\sigma_{p} / \bar{p}$ behave very $y_{737}$ differently: the stochastic pressure forcing is more prone $\mathrm{e}_{738}$ to trigger strong chaotic radius fluctuations than its si-739 nusoidal counterpart.

In this work, we have demonstrated that stochastic 741 forcing can induce interesting and unexpected bubble be-742 haviors, presumably induced by the strongly non-linear ${ }_{743}$ nature of the bubble oscillator. This paves the way to744 study other type of noises (e.g., dichotomous or shot noises) and to investigate how random forcing could be conveniently exploited in various applications. For $\mathrm{ex}^{-745}$ ample, noise-induced violent cavities implosions - attained when intensity and correlation of pressure fluctuations are high - can be used to make water disinfection ${ }^{746}$ processes based on hydrodynamic cavitation and sono- ${ }^{748}$ chemical reactions more energy efficient.

\section{ACKNOWLEDGMENT}

RV, CM and LR are grateful for the partial funding to this work provided by the European Union (Project $_{755}$ BioEnPro4To POR FESR 2014/2020).

\section{Appendix A: Role of correlation time}

In order to elucidate the increment of variability of 761 $R(t)$ with $\tau_{p}$, Panels $7 a, b$ report some exemplifying por-762 tions of time-series $R(t) / R_{e q}$ obtained with the samer6s noise intensity $\sigma_{p} / \bar{p}=0.70$ (chosen in order to be in the764 chaos domain) but different noise time-scales, namely765 $\tau_{p}=T_{n} / 2$ and $\tau_{p}=2 T_{n}$, respectively. To relate the bub-766 ble radius dynamics to the pressure fluctuations, red dots767 plotted in Panels $7 a, b$ (along the line $R(t) / R_{e q}=1$ ) mark768 the instants when the instantaneous forcing pressure is769 below average (i.e., $p(t)<\bar{p}$ ). From a physical point of 770 view, when the instantaneous pressure is below average 771 the bubble radius tends to increase; on the contrary, ra-772 dius contractions are promoted when the instantaneous773 pressure is above average (i.e., $p(t)>\bar{p}$, identified by not74 dots at $\left.R(t) / R_{e q}=1\right)$.
Panels $7 a$ shows that, when the correlation time of the pressure forcing, $\tau_{p}$, is low, time segments with pressure below average $(p(t)<\bar{p})$ and time segments with pressure above average $(p(t)>\bar{p})$ alternate fairly regularly: the red dots plotted at $R(t) / R_{e q}=1$ are grouped in short time segments, and are followed by short segments where no dots are reported. A key consequence of short time segments with pressure below average $(p(t)<\bar{p})$ is that the bubble radius cannot attain large increments (see the black time-series in Panel 7a).

In contrast, for high values of the correlation time, time segments with pressure below average $(p(t)<\bar{p})$ persist for long time and are followed by long-lasting time intervals with pressure values above average $(p(t)>\bar{p})$ : Fig. $7 b$ shows, indeed, that long sets of red dots alternate with long sets without dots. In this case, time segments in which the pressure is below average $(p(t)<\bar{p})$ last so long that very large radius increments are attained (e.g., see the strong growth of $R(t)$ occurring at $t / T_{n} \approx 255$ in the second time segments of Panel 7b). Vice versa, when the condition $p(t)>\bar{p}$ is restored, the bubble collapses. As explained in Section III B, the occurrence of these phases of remarkable radius expansion contributes to trigger the irregularity of $R(t)$.

\section{Appendix B: Statistical analysis - pdf and cdf}

In Fig. 8, we show some exemplifying cases, in order to discuss the effect of $\sigma_{p} / \bar{p}$ and $\tau_{p}$ on the the probability density function (pdf) and cumulative density function (cdf) of the bubble radius. To this end, it is useful to define a benchmark case (see the thick black lines).

We selected the benchmark correlation time $\tau_{p}=T_{n}$. This choice was based on past studies that considered sinusoidal pressure oscillations. These studies found that complex dynamics occurs when the period of the sinusoidal forcing is equal to the natural oscillation period of the bubble $[13,25,41,42,45]$. Therefore, we expect bubbles to exhibit interesting dynamics when the correlation time of the noise signal is equal to the natural oscillation period of the bubble. On the other hand, we selected the benchmark noise intensity $\sigma_{p} / \bar{p}=0.60$. This choice was based on the results reported in Figs. $2 a, b$, showing chaotic dynamics of the bubble radius in the $\sigma_{p} / \bar{p}$-range $[0.30,1.10]$. We wanted to focus on bubble exhibiting a chaotic behavior, so we chose a value of noise intensity in this chaos range.

The noise intensity (in terms of $\sigma_{p} / \bar{p}$ ) was then altered, keeping $\tau_{p}=T_{n}$ (broken lines in Panels $8 a, c, d$ ). Finally, $\tau_{p}$ was also changed while $\sigma_{p} / \bar{p}$ was kept at its benchmark value (broken lines in Panel $8 b$ ). The dotted (dash-dot) lines refer to a parameter higher (lower) than the benchmark value.

Irrespectively of the noise parameters $\left\{\sigma_{p} / \bar{p}, \tau_{p}\right\}$, the quantity $R / R_{e q}$ exhibits a unimodal pdf (Panels $8 a, b$ ), whose shape, though, depends significantly on the noise intensity (Fig. 8a). In particular, increments of $\sigma_{p} / \bar{p}$ 

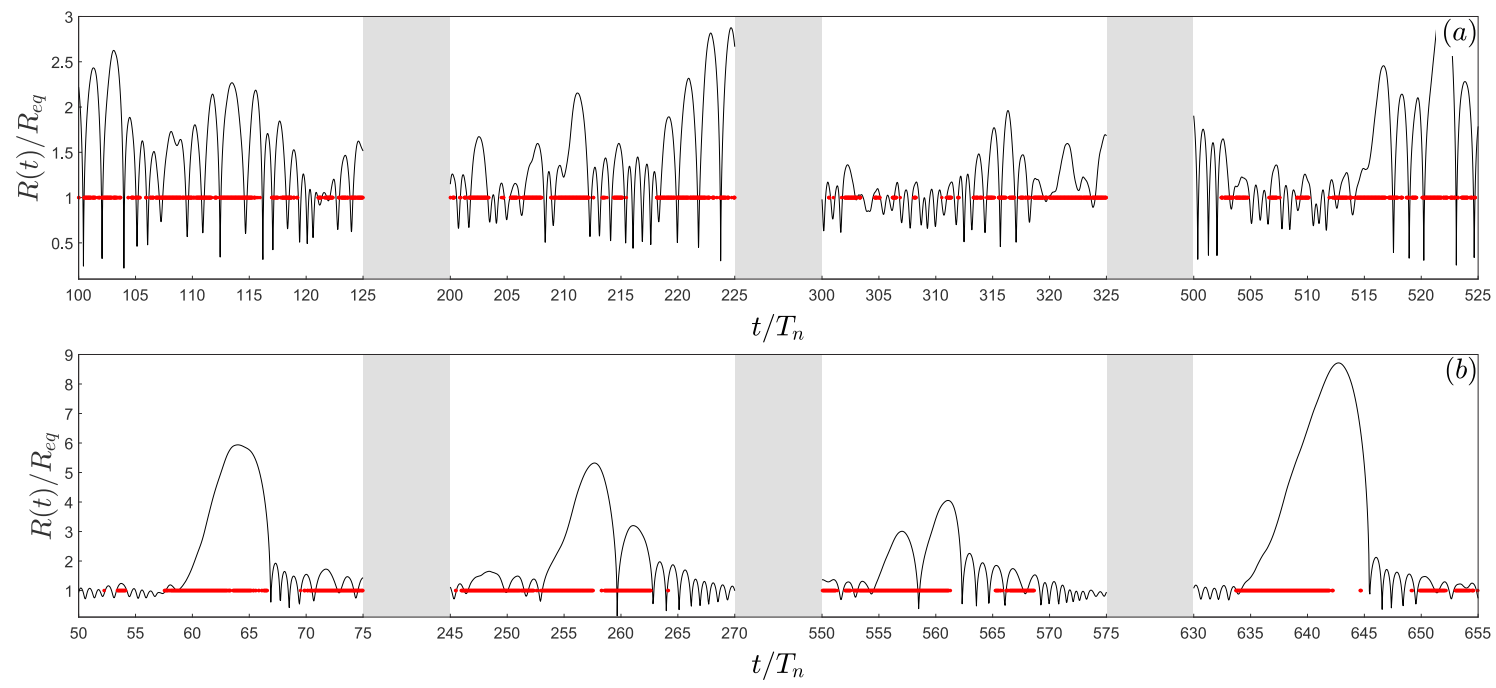

FIG. 7. Time-series of $R(t) / R_{e q}$ in four relevant time segments in the case of $\tau_{p}=T_{n} / 2(a)$ and $\tau_{p}=2 T_{n}(b)$. In both cases $\sigma_{p} / \bar{p}=0.70$. The red dots plotted at $R(t) / R_{e q}=1$ mark the instants when $p(t)<\bar{p}$, and should not be confused with the dynamics of $R(t) / R_{e q}$ reported by the black line. Panels $(a)$ and (b) report different ranges in the vertical axis.
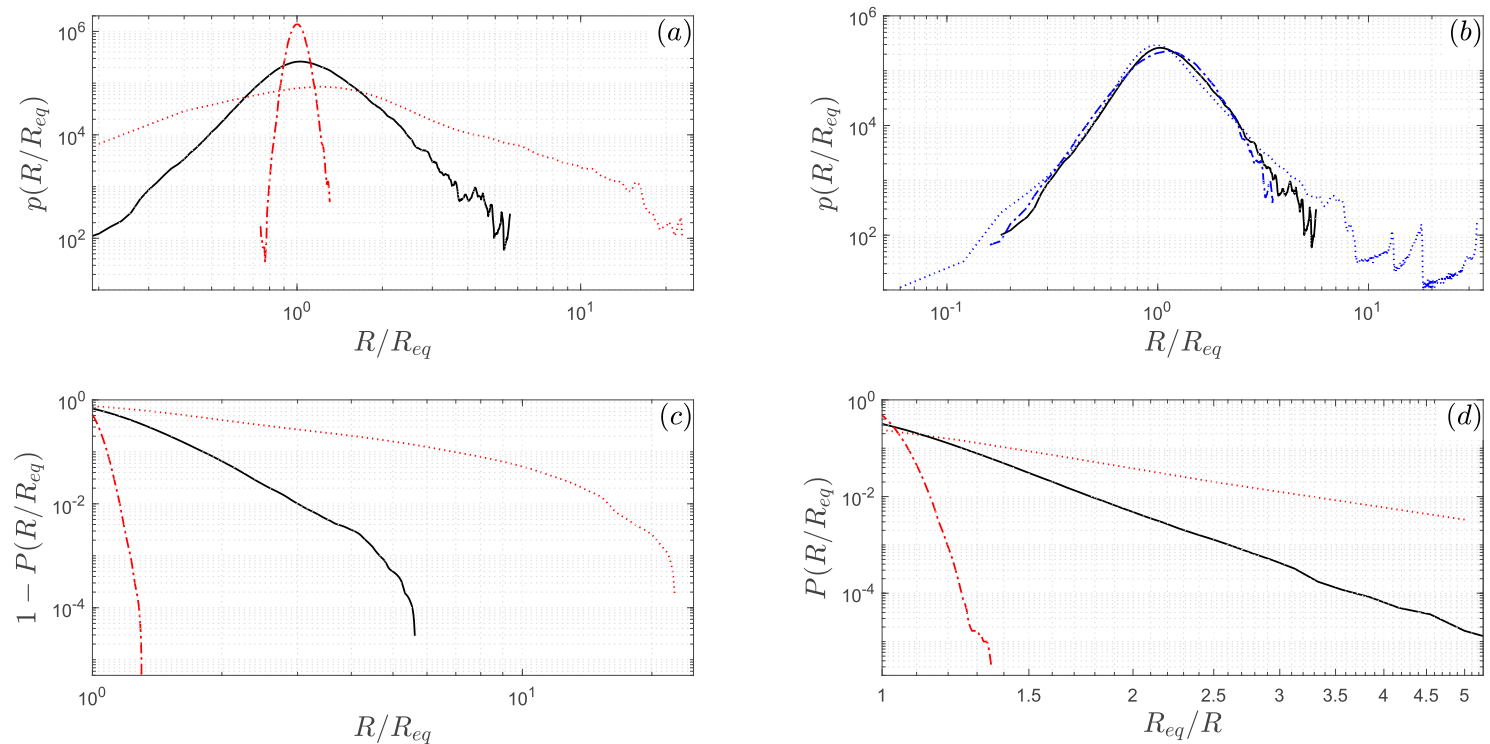

$$
\begin{gathered}
- \text { Benchmark }\left(\sigma_{p} / \bar{p}=0.60, \tau_{p}=T_{n}\right) \\
--\sigma_{p} / \bar{p}=0.14, \tau_{p}=T_{n} \quad--\sigma_{p} / \bar{p}=0.60, \tau_{p}=0.5 T_{n} \\
\cdots \cdots \sigma_{p} / \bar{p}=1.10, \tau_{p}=T_{n}
\end{gathered}
$$

FIG. 8. ( $(a, b)$ Probability density function of the metric $R / R_{e q}$. (c) Complementary cumulative distribution function of $R / R_{e q}$ evaluated for $R / R_{e q}>1$ (right tail of the distribution). (d) Cumulative distribution function of $R / R_{e q}$ evaluated for $R / R_{e q}<1$ (left tail of the distribution), note that the horizontal axis reports $R_{e q} / R$ and not $R / R_{e q}$ as in Panel $(c)$.

induce the reduction of the peak height, the fattening of ${ }_{785}$ the tails, more asymmetrical pdfs, and the increment of $f_{786}$ the mode. Differently from $\sigma_{p} / \bar{p}$, changes of $\tau_{p}$ induce $_{787}$ less relevant effects (Fig. 8b). No changes of the peak ${ }_{788}$ height, of the mode of the pdf, and of the symmetry of $f_{789}$ the curves are in fact observed. The only relevant effect is $_{790}$ a slight expansion of the distribution range toward higher ${ }_{791}$ values of $R / R_{e q}$, which occurs when the correlation time ${ }_{792}$ increases (see the right tail of the dotted curve in Fig. ${ }^{-793}$
$8 b)$.

The tails are better described by the cumulative distribution functions. A complementary distribution is adopted to analyze the right tail, (see Fig. 8c). In order to focus on the left tail, the cumulative distribution is evaluated (see Fig. 8d). Increments of the noise intensity mainly induce a fattening of the tails and an increment of the range (see Panels $8 c, d$ ). In the right tail, the range increases from 2 to 20 when $\sigma_{p} / \bar{p}$ increases from 
0.14 to 1.10. Moreover, the frequency of occurrence of 809 a given $R / R_{e q}$ changes of orders of magnitude, for the same increment of $\sigma_{p} / \bar{p}$. The same behavior is observed in the left tail: the minimum value attained by $R / R_{e q}$ reduces from 0.6 to 0.2 , when $\sigma_{p} / \bar{p}$ increases from $0.14^{810}$ to 0.60 . Interestingly, the further increment of $\sigma_{p} / \bar{p}$ from 0.60 to 1.1 does not lead to a reduction of $R / R_{e q}$. Thes11 distribution does not extend beyond $0.2\left(R_{e q} / R=5\right.$ in ${ }_{812}$ Fig. $8 d)$. However, the frequency of occurrence of thiss13 extreme value increases of more than one order of magni-814 tude. Finally, as surmised from the analysis of Panels $8 a_{815}$ and $8 b$, the pdfs of $R / R_{e q}$ display asymmetry. In fact the 816 right tail is always characterized by a power-law behavior ${ }_{817}$ (linear in the log-log diagrams of Panel $8 c$ ) for low valuessis of $R / R_{e q}$ followed by a cut-off. On the contrary, the leftsig tail is always approximately linear (Panel $8 d$ ).

\section{Appendix C: Numerical Details}

In order to evaluate the response of a gas bubble to a pressure forcing, the numerical integration of (1) is required. To this aim, the dimensional Eq. (1) is firstly made dimensionless adopting the length scale $R_{e q}$ (i.e., the bubble radius in equilibrium conditions) and the time scale $T_{n}$ (i.e., the period of bubble free oscillations, see Eq. 5). Secondly, the second-order differential dimensionless equation is transformed in the system of two first-order differential dimensionless equations

$$
\left\{\begin{array}{l}
\tilde{y}_{2}=\frac{\mathrm{d} \tilde{y}_{1}}{\mathrm{~d} \tilde{t}} \\
\frac{\mathrm{d} \tilde{y}_{2}}{\mathrm{~d} \tilde{t}}=\frac{\frac{p_{w}-p(\tilde{t})}{\mathcal{P} \tilde{y}_{1}}+\frac{\tilde{y}_{2}}{\mathcal{N} \tilde{y}_{1}}\left[p_{G}(1-3 k)-p(\tilde{t})+p_{v}\right]-\frac{1}{\mathcal{N}} \frac{\mathrm{d} p(\tilde{t})}{\mathrm{d} \tilde{t}}-\left(1-\frac{\mathrm{Ma}}{3}\right) \frac{3 \tilde{y}_{2}^{2}}{2 \tilde{y}_{1}}}{1-\mathrm{Ma}+\frac{4 \mu}{\mathcal{M} \tilde{y}_{1}}},
\end{array}\right.
$$

where tilde denotes dimensionless quantities, $\tilde{y}_{1}={ }_{843}$ $R / R_{e q}, \mathrm{Ma}=\tilde{y}_{2} R_{e q} / c T_{n}$ is the Mach number, $\mathcal{P}={ }_{844}$ $\rho R_{\text {eq }}^{2} / T_{n}^{2}, \mathcal{M}=c \rho / R_{e q}$, and $\mathcal{N}=\mathcal{M} / T_{n}$. Finally, $p_{w^{845}}$ and $p_{G}$ can be expressed, according to (2-4), in terms of $f_{846}$ $\tilde{y}_{1}$ and $\tilde{y}_{2}$ as

$$
p_{G}=\left(\frac{2 S}{R_{e q}}-p_{v}+\bar{p}\right)\left(\frac{1}{\tilde{y}_{1}}\right)^{3 k},
$$

$$
p_{w}=p_{G}+p_{v}-\frac{2 S}{R_{e q} \tilde{y}_{1}}-\frac{4 \mu \tilde{y}_{2}}{T_{n} \tilde{y}_{1}} .
$$

The system of equation $(\mathrm{C} 1)$ was numerically solved ${ }^{854}$ by an explicit Runge-Kutta approach by using the ${ }^{855}$ Dormand-Prince pair [68].

In order to select the appropriate time-step for numer- ${ }^{857}$ ical integration, a sensitivity analysis about this param- ${ }^{858}$ eter was performed. The test case was a gas bubble with ${ }^{859}$ $R_{e q}=5 \mu \mathrm{m}, R(0) / R_{e q}=2$ and $\dot{R}(0)=0$ in a uniform ${ }^{860}$ pressure field. Three time steps $\left(\Delta t=\left[10^{-7}, 10^{-8}, 10^{-9}\right]^{661}\right.$ s) were tested in the numerical simulations of the bubble 862 dynamics (see Fig. 9). Panel $9 a$ shows that $\Delta t=10^{-9}{ }_{863}$ $\mathrm{s}$ and $\Delta t=10^{-8} \mathrm{~s}$ led to a bubble response (in terms 864 of $R(t)$ ) indistinguishable, while $\Delta t=10^{-7} \mathrm{~s}$ led to $\mathrm{a}_{865}$ less precise simulation of the system dynamics. To bet-866 ter quantify the quality of the numerical integrations, we 867 evaluated the relative error

$$
\varepsilon_{R}(t)=\frac{\left\|R(\Delta t, t)-R_{\mathrm{REF}}(t)\right\|}{R_{\mathrm{REF}}(t)},
$$

where $R(\Delta t, t)$ is the bubble radius at the instant $t$ eval-872 uated with a numerical simulation in which the time-step 873
$\Delta t$ was adopted. The term $R_{\mathrm{REF}}(t)$ is the "exact" reference value. In this case, we adopted $R_{\mathrm{REF}}(t)=R(\Delta t=$ $\left.10^{-9}, t\right)$. The time step $\Delta t=10^{-8} \mathrm{~s}$ was found suitable for the numerical integrations, as the maximum error $\varepsilon \sim 0.02$ was attained (see Fig. $9 b$ ).

In order to guarantee that the statistical description of a stochastic process was significant, two tests were performed. The first test concerns the duration of the considered stochastic process. In particular, we studied whether the same statistical values were obtained, irrespectively of the length of the analyzed time-series. Fig. 10 reports the behavior of two statistical metrics as a function of $\sigma_{p} / \bar{p}$, as already discussed in Fig. 4. Each statistical index was evaluated from four time-series, $R(t)$, characterized by different durations, $T$. It can be observed that simulations carried out with $T>2000 T_{n}$ lead to curves characterized by the same behavior. The duration $T=4000 T_{n}$ was therefore deemed appropriate for the statistical analysis of the stochastic bubble dynamics.

The second test was to verify the independence of the results from a single realization. Namely, whether different stochastic realizations of the process lead to the same statistical indexes. Fig. 11 reports two statistical parameters of Fig. 4. Each statistical index was evaluated with seven time-series, $R(t)$, characterized by a different pressure forcing. Each pressure time-series was characterized by the same statistics $\left(\sigma_{p}, \tau_{p}\right)$, but a different set of random numbers (see Eq. 7) was adopted to introduce randomness. It can be observed that all simulations give curves characterized by the same behavior. Moreover, the mean value, the standard deviation and the kurtosis 

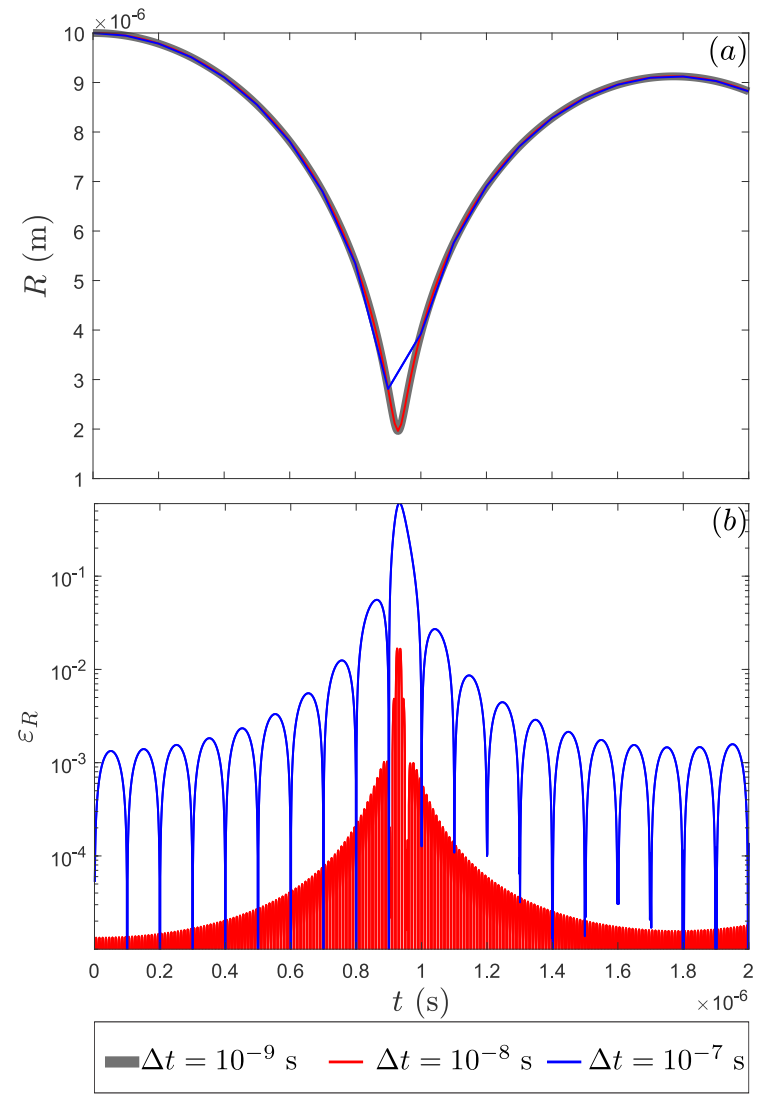

FIG. 9. (a) Example of curves $R(\Delta t, t)$ numerically computed adopting different time-steps $\Delta t$. (b) Relative error $\varepsilon_{R}(t)$ occurring in the numerical computation performed with different time-steps. The relative error is evaluated considering the curve computed with $\Delta t=10^{-9} \mathrm{~s}$ the exact reference. The initial conditions are $R(0) / R_{e q}=2$ and $\dot{R}(0)=0$. The pressure field is uniform.
${ }_{874}$ of the bubble radius were basically the same.
[1] F. Risso, Agitation, mixing, and transfers induced by 896 bubbles, Ann. Rev. Fluid Mech. 50, 25 (2018).

[2] A. Prosperetti, $\quad$ Vapor ${ }^{897}$ Ann. Rev. Fluid Mech. 49, 221 (2017). 899

[3] C. Brennen, Cavitation and bubble dynamics (Cam-900 bridge University Press, 2013) pp. 1-249.

901

[4] M. Azmin, C. Harfield, Z. Ahmad, M. Edirisinghe, and902 E. Stride, How do microbubbles and ultrasound inter-903 act? Basic physical, dynamic and engineering principles,904 Curr. Pharm. Des. 18, 2118 (2012).

[5] Y. Hao and A. Prosperetti, The effect of viscosity906 on the spherical stability of oscillating gas bubbles,907 Phys Fluids 11, 1309 (1999).

[6] J.-L. Laborde, C. Bouyer, J.-P. Caltagirone, and909 A. Grard, Acoustic bubble cavitation at low frequencies,910 Ultrasonics 36, 589 (1998).

[7] A Prosperetti, The thermal behaviour of oscillating gasis bubbles, J. Fluid Mech. 222, 587 (1991).

[8] I. Rayleigh, On the pressure developer uid during the collapse of a spherical cavity, 915 Phil. Mag.Ser. 6 34, 94 (1917).
[9] M. Plesset, The dynamics of cavitation bubbles, J. Appl. Mech. 16, 277 (1949).

[10] F. R. Gilmore, The growth or collapse of a spherical bubble in a viscous compressible liquid, Report No 26-4 Hydrodynamics Laboratory, California Institute of Technology, Pasadena, California, USA (1952).

[11] M. S. Plesset and A. Prosperetti, Bubble dynamics and cavitation, Ann. Rev. Fluid Mech. 9, 145 (1977).

[12] A. Prosperetti, Bubble dynamics in a compressible liquid. Part 1. First-order theory, J. Fluid Mech. 168, 457 (1986).

[13] W. Lauterborn and T. Kurz, Physics of bubble oscillations, Rep. Prog. Phys. 73 , 10.1088/0034-4885/73/10/106501 (2010).

[14] H. Lin, B. Storey, and A. Szeri, Inertially driven inhomogeneities in violently collapsing bubbles: The validity of the Rayleigh-Plesset equation, J. Fluid Mech. 452, 145 (2002).

[15] A. Moshaii and R. Sadighi-Bonabi, Role of liquid compressional viscosity in the dynamics of a sonoluminescing bubble, Phys Rev E. 70, 6 (2004). 

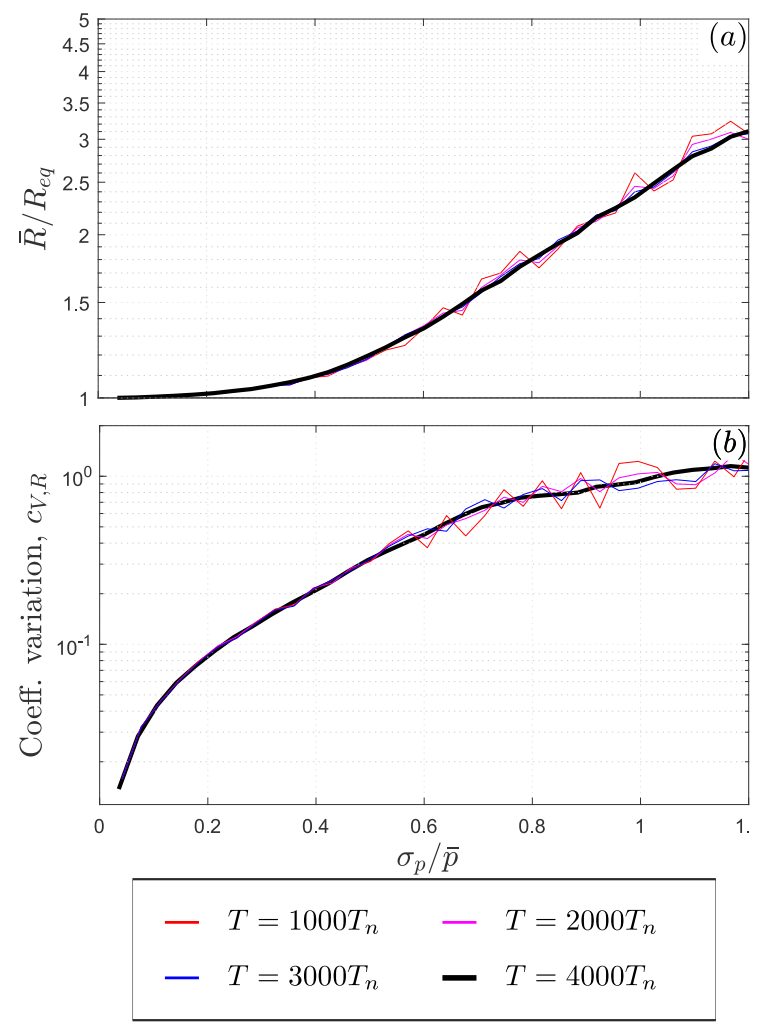

FIG. 10. Effect of the duration $T$ of the simulation on the statistical metrics that describe the time-series $R(t)$. Similar to Fig. 4, two statistical parameters and their dependence on $\sigma_{p} / \bar{p}$ are considered. The different curves were evaluated considering different length of the simulation. The parameter $\tau_{p}=T_{n}$ is adopted.

[16] O. Supponen, D. Obreschkow, and M. Farhat, Rebounds947 of deformed cavitation bubbles, Phys. Rev. Fluids 3,948 10.1103/PhysRevFluids.3.103604 (2018).

[17] M. Ghorbani, O. Oral, S. Ekici, D. Gozuacik, and950 A. Kosar, Review on lithotripsy and cavitation in urinary951 stone therapy, IEEE Rev. Biomed. Eng. 9, 264 (2016). 952

[18] V. Agnese, V. Costa, G. Scoarughi, C. Corso, V. Ca-953 rina, A. De Luca, D. Bellavia, L. Raimondi, S. Pa-954 gani, M. Midiri, G. Stassi, R. Alessandro, M. Fini,955 G. Barbato, and G. Giavaresi, Focused ultrasound ef-956 fects on osteosarcoma cell lines, BioMed Res. Int. 2019,957 $10.1155 / 2019 / 6082304$ (2019)

[19] M. Dular, T. Griessler-Bulc, I. Gutierrez-Aguirre,959 E. Heath, T. Kosjek, A. Krivograd Klemeni, M. Oder,960 M. Petkovek, N. Raki, M. Ravnikar, A. arc, B. irok,961 M. Zupanc, M. itnik, and B. Kompare, Use of962 hydrodynamic cavitation in (waste)water treatment,963 Ultrason. Sonochem. 29, 577 (2016).

[20] A. Šarc, M. Oder, and M. Dular, Can rapid965 pressure decrease induced by supercavitation effi-966 ciently eradicate Legionella pneumophila bacteria?,967 Desalin. Water Treat. 57, 2184 (2016).

[21] J. Carpenter, M. Badve, S. Rajoriya, S. George, V. Saha-969 ran, and A. Pandit, Hydrodynamic cavitation: An emerg-970 ing technology for the intensification of various chemicalo71 and physical processes in a chemical process industry,972
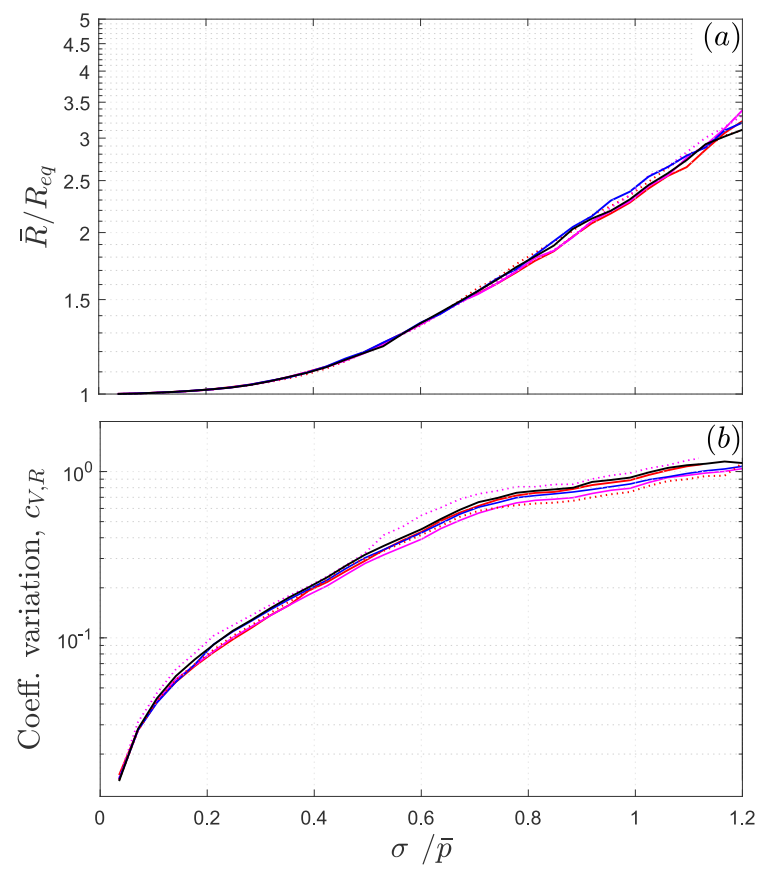

FIG. 11. Effect of different realizations of the stochastic process on the statistical parameters that describe the timeseries $R(t)$. Similar to Fig. 4, two statistical parameters and their dependence on $\sigma_{p} / \bar{p}$ are considered. The different curves were evaluated with the same noise intensities and correlation times, but with a different set of random numbers. The parameter $\tau_{p}=T_{n}$ is adopted.

Rev. Chem. Eng. 33, 433 (2017).

[22] E. Burzio, F. Bersani, G. Caridi, R. Vesipa, L. Ridolfi, and C. Manes, Water disinfection by orificeinduced hydrodynamic cavitation, Ultrason. Sonochem. 60, 10.1016/j.ultsonch.2019.104740 (2020).

[23] G.-F. Li, M.-Q. Cao, H.-L. Chen, and C.-Z. Ni, Modeling air gun signatures in marine seismic exploration considering multiple physical factors, Appl. Geophys. 7, 158 (2010).

[24] Y. Liu, A.-M. Zhang, Z. Tian, and S. Wang, Investigation of free-field underwater explosion with eulerian finite element method, Ocean Eng. 166, 182 (2018).

[25] F. Hegedus and K. Klapcsik, The effect of high viscosity on the collapse-like chaotic and regular periodic oscillations of a harmonically excited gas bubble, Ultrason. Sonochem. 27, 153 (2015).

[26] S. Popinet and S. Zaleski, Bubble collapse near a solid boundary: A numerical study of the influence of viscosity, J. Fluid Mech. 464, 137 (2002).

[27] E. Zwaan, S. Le Gac, K. Tsuji, and C.-D. Ohl, Controlled cavitation in microfluidic systems, Phys Rev Lett 98, 10.1103/PhysRevLett.98.254501 (2007).

[28] Q. Wang, Multi-oscillations of a bubble in a compressible liquid near a rigid boundary, J. Fluid Mech. 745, 509 (2014).

[29] A. Doinikov and A. Bouakaz, Theoretical model for coupled radial and translational motion of two bubbles at arbitrary separation distances, Phys. Rev. E 92, 10.1103/PhysRevE.92.043001 (2015).

[30] Y. Fan, H. Li, J. Zhu, and W. Du, A simple model of 
bubble cluster dynamics in an acoustic field, Ultrason1036 Sonochem. 64, 10.1016/j.ultsonch.2019.104790 (2020). 1037

[31] C. Kling and F. Hammitt, A photographic studyoз of spark-induced cavitation bubble collapse10з9 J Fluids Eng Trans ASME 94, 825 (1972).

[32] I. Akhatov, O. Lindau, A. Topolnikov, R. Met+041 tin, N. Vakhitova, and W. Lauterborn, Collapse042 and rebound of a laser-induced cavitation bubble1043 Phys. Fluids 13, 2805 (2001).

[33] J. Rensen, D. Bosman, J. Magnaudet, C.-D. Ohl,1045 A. Prosperetti, R. Tgel, M. Versluis, and D. Lohse, Spi 4046 raling bubbles: How acoustic and hydrodynamic forces 047 compete, Phys Rev Lett 86, 4819 (2001).

34] A. Bergant, A. Simpson, and A. Tijsseling, Waterous hammer with column separation: A historical review1050 J. Fluids Struct. 22, 135 (2006).

[35] A. Prosperetti, Bubble phenomena in sound fields: parto52 one, Ultrasonics 22, 69 (1984).

[36] T. Leighton, From seas to surgeries, from babbling brooks054 to baby scans: The acoustics of gas bubbles in liquids1055 Int. J. Mod. Phys. B 18, 3267 (2004).

[37] P. Kumar and A. Pandit, Modeling hydrodynamic cavit057 tation, Chem Eng Technol 22, 1017 (1999).

[38] S. Arrojo and Y. Benito, A theoretical study of hydrody 4059 namic cavitation, Ultrason. Sonochem. 15, 203 (2008). 1060

[39] W. Lauterborn, T. Kurz, R. Mettin, and C. Ohl, Exper ${ }^{4} 061$ imental and theoretical bubble dynamics, Adv. Chem1062 Phys. 110, 295 (1999).

[40] J. Keller and M. Miksis, Bubble oscillations of large amł064 plitude, J. Acoust. Soc. Am. 68, 628 (1980).

[41] W. Lauterborn and E. Cramer, Subhar ${ }^{1066}$ monic route to chaos observed in acoustics1067 Phys Rev Lett 47, 1445 (1981).

[42] U. Parlitz, V. Englisch, C. Scheffczyk, and W. Lauter ${ }^{0} 069$ born, Bifurcation structure of bubble oscillators1070 J. Acoust. Soc. Am. 88, 1061 (1990).

[43] T. Leighton, Bubble population phenomena in acoustico72 cavitation, Ultrason. Sonochem. 2, S123 (1995). 1073

[44] G. Simon, P. Cvitanovi, M. Levinsen, I. Csabai, ${ }_{1074}$ and . Horvth, Periodic orbit theory applied to075 a chaotically oscillating gas bubble in water 1076 Nonlinearity 15, 25 (2002).

[45] C. Macdonald and J. Gomatam, Chaotic dy 7078 namics of microbubbles in ultrasonic fields1079 Proc. Inst. Mech. Eng. Part C J. Mech. Eng. Sci. 220, 383

[46] V. Moholkar, S. Rekveld, and M. Warmoeskerken, Mod 4081 eling of the acoustic pressure fields and the distributionos of the cavitation phenomena in a dual frequency sonicoss processor, Ultrasonics 38, 666 (2000).

[47] P. Kanthale, A. Brotchie, M. Ashokkumar, and085 F. Grieser, Experimental and theoretical investigation\$086 on sonoluminescence under dual frequency conditions1087 Ultrason. Sonochem. 15, 629 (2008).

[48] Y. Zhang and Y. Zhang, Chaotic oscillations of 089 gas bubbles under dual-frequency acoustic excitation1090 Ultrason. Sonochem. 40, 151 (2018).

[49] T. Leighton, Transient excitation of insonated bubblesiog2 Ultrasonics 27, 50 (1989).

[50] K. Efthymiou, N. Pelekasis, M. Butler, D. Thomas1094 and V. Sboros, The effect of resonance on trant095 sient microbubble acoustic response: Experiı096 mental observations and numerical simulations, J. Acoust. Soc. Am. 143, 1392 (2018).
[51] E. Igualada-Villodre, A. Medina-Palomo, P. VegaMartnez, and J. Rodrguez-Rodrguez, Transient effects in the translation of bubbles insonated with acoustic pulses of finite duration, J. Fluid Mech. 836, 649 (2018).

[52] N. Berglund and B. Gentz, Noise-Induced Phenomena in Slow-Fast Dynamical Systems: A Probability and its Applications (Springer London, 2006).

[53] F. Sagus, J. Sancho, and J. Garca-Ojalvo, Spatiotemporal order out of noise, Rev. Mod. Phys. 79, 829 (2007).

[54] L. Ridolfi, P. D'Odorico, and F. Laio, Noise-Induced Phenomena in the Environmental Sciences (Cambridge University Press, 2011).

[55] M. Freidlin, J. Szucs, and A. Wentzell, Random Perturbations of Dynamical Systems, Grundlehren der mathematischen Wissenschaften (Springer New York, 2012).

[56] W. McNamara III, Y. Didenko, and K. Suslick, Sonoluminescence temperatures during multi-bubble cavitation, Nature 401, 772 (1999).

[57] V. Moholkar and A. Pandit, Bubble behavior in hydrodynamic cavitation: Effect of turbulence, AIChE J. 43, 1641 (1997).

[58] A. Sharma, P. Gogate, A. Mahulkar, and A. Pandit, Modeling of hydrodynamic cavitation reactors based on orifice plates considering hydrodynamics and chemical reactions occurring in bubble, Chem. Eng. J. 143, 201 (2008).

[59] G. Uhlenbeck and L. Ornstein, On the theory of the Brownian motion, Physical Review 36, 823 (1930).

[60] A. Li and G. Ahmadi, Dispersion and deposition of spherical particles from point sources in a turbulent channel flow, Aerosol Sci. Technol. 16, 209 (1992).

[61] P. Jung, Periodically driven stochastic systems, Phys. Rep. 234, 175 (1993).

[62] E. Codling, M. Plank, and S. Benhamou, Random walk models in biology, J. R. Soc. Interface 5, 813 (2008).

[63] A. Gu, B. Guo, and B. Wang, Long term behavior of random Navier-Stokes equations driven by colored noise, Discrete Contin. Dyn. Syst. Ser. B 25, 2495 (2020).

[64] R. Mettin and A. Doinikov, Translational instability of a spherical bubble in a standing ultrasound wave, Appl. Acoust. 70, 1330 (2009).

[65] E. Neppiras, Acoustic cavitation, Phys. Rep. 61, 159 (1980).

(260)6F. Hegedus, S. Koch, W. Garen, Z. Pandula, G. Pal, L. Kullmann, and U. Teubner, The effect of high viscosity on compressible and incompressible Rayleigh-Plesset-type bubble models, Int. J. Heat Fluid Flow 42, 200 (2013).

[67] D. Gillespie, Exact numerical simulation of the Ornstein-Uhlenbeck process and its integral, Phys Rev E. 54, 2084 (1996).

[68] J. Dormand and P. Prince, A family of embedded RungeKutta formulae, J. Comput. Appl. Math. 6, 19 (1980).

[69] H. Nazari-Mahroo, K. Pasandideh, H.A. Navid and R. Sadighi-Bonabi, Influence of liquid compressibility on the dynamics of single bubble sonoluminescence, Phys. Lett. A 382, 1962 (2018).

[70] R. Löfstedt, B.P. Barber, and S.J. Putterman, Toward a hydrodynamic theory of sonoluminescence, Phys. Fluids 5, 2911-2928 (1992). 